\title{
Identification and selection rules of the spin-wave eigenmodes in a normally magnetized nanopillar
}

\author{
V. V. Naletov, ${ }^{1,2}$ G. de Loubens, ${ }^{1}$ G. Albuquerque, ${ }^{3}$ S. Borlenghi, ${ }^{1}$ V. Cros,${ }^{4}$ G. Faini, ${ }^{5}$ J. Grollier, ${ }^{4}$ H. Hurdequint, ${ }^{6}$ \\ N. Locatelli, ${ }^{4}$ B. Pigeau, ${ }^{1}$ A. N. Slavin, ${ }^{7}$ V. S. Tiberkevich, ${ }^{7}$ C. Ulysse, ${ }^{5}$ T. Valet, ${ }^{3}$ and O. Klein ${ }^{1, *}$ \\ ${ }^{1}$ Service de Physique de l'État Condensé (CNRS URA 2464), CEA Saclay, FR-91191 Gif-sur-Yvette, France \\ ${ }^{2}$ Physics Department, Kazan Federal University, Kazan 420008, Russian Federation \\ ${ }^{3}$ In Silicio, 730 rue René Descartes FR-13857 Aix En Provence, France \\ ${ }^{4}$ Unité Mixte de Physique CNRS/Thales and Université Paris Sud 11, RD 128, FR-91767 Palaiseau, France \\ ${ }^{5}$ Laboratoire de Photonique et de Nanostructures, Route de Nozay FR-91460 Marcoussis, France \\ ${ }^{6}$ Laboratoire de Physique des Solides, Université Paris-Sud, FR-91405 Orsay, France \\ ${ }^{7}$ Department of Physics, Oakland University, Michigan 48309, USA
}

(Received 21 July 2011; revised manuscript received 5 December 2011; published 21 December 2011)

\begin{abstract}
We report on a spectroscopic study of the spin-wave eigenmodes inside an individual normally magnetized two-layer circular nanopillar (permalloy|copper|permalloy) by means of a magnetic resonance force microscope. We demonstrate that the observed spin-wave spectrum critically depends on the method of excitation. While the spatially uniform radio-frequency (rf) magnetic field excites only the axially symmetric modes having azimuthal index $\ell=0$, the rf current flowing through the nanopillar, creating a circular rf Oersted field, excites only the modes having azimuthal index $\ell=+1$. Breaking the axial symmetry of the nanopillar, either by tilting the bias magnetic field or by making the pillar shape elliptical, mixes different $\ell$-index symmetries, which can be excited simultaneously by the rf current. Experimental spectra are compared to theoretical prediction using both analytical and numerical calculations. An analysis of the influence of the static and dynamic dipolar coupling between the nanopillar magnetic layers on the mode spectrum is performed.
\end{abstract}

DOI: $10.1103 /$ PhysRevB.84.224423

PACS number(s): 76.50.+g, 85.75.-d, 75.30.Ds, 78.47.-p

\section{INTRODUCTION}

Technological progress in the fabrication of hybrid nanostructures using magnetic metals has allowed the emergence of a new science aimed at utilizing spin-dependent effects in the electronic transport properties. ${ }^{1}$ An elementary device of spintronics consists of two magnetic layers separated by a normal layer. It exhibits the well-known giant magnetoresistance (GMR) effect; ${ }^{2,3}$ that is, its resistance depends on the relative angle between the magnetic layers. Nowadays, this useful property is extensively used in magnetic sensors. ${ }^{4,5}$ The converse effect is that a direct current can transfer spin angular momentum between two magnetic layers separated by either a normal metal or a thin insulating layer. ${ }^{6,7}$ As a result, a spin polarized current leads to a very efficient destabilization of the orientation of a magnetic moment. ${ }^{8}$ Practical applications are the possibility to control the digital information in magnetic random access memories (MRAMs) ${ }^{9,10}$ or to produce high-frequency signals in spin-transfer nano-oscillators (STNOs). ${ }^{11,12}$

From an experimental point of view, the precise identification of the spin-wave (SW) eigenmodes in hybrid magnetic nanostructures remains to be done. ${ }^{13-18}$ Of particular interest is the exact nature of the modes excited by a current perpendicular to plane in STNOs. Here the identification of the associated symmetry behind each mode is essential. It gives a fundamental insight about their selection rules and about the mutual coupling mechanisms that might exist intra- or

Published by the American Physical Society under the terms of the Creative Commons Attribution 3.0 License. Further distribution of this work must maintain attribution to the author(s) and the published article's title, journal citation, and DOI.
inter-STNOs. It also determines the optimum strategy to couple to the auto-oscillating mode observed when the spintransfer torque compensates the damping, which is vital knowledge to achieve phase synchronization in arrays of nanopillars. ${ }^{19}$ These SW modes also have a fundamental influence on the high-frequency properties of these devices and in particular on the noise of magnetoresistive sensors. ${ }^{20,21}$

A natural mean to probe SW modes in hybrid nanostructures is to use their magnetoresistance properties. For instance, thermal SW can be directly detected in the noise spectrum of tunneling magnetoresistance (TMR) devices owing to their large TMR ratio. ${ }^{22,23}$ It is also possible to use spintorque-driven ferromagnetic resonance (ST-FMR). ${ }^{24-30}$ In this approach, an rf current flowing through the magnetoresistive device is used to excite the precession of magnetization and to detect it through a rectification effect. Direct excitation of SW modes by the rf field generated by microantennas and their detection through dc rectification ${ }^{31}$ or high-frequency GMR measurements ${ }^{32}$ has also been reported in spin-valve sensors. In all these experiments, the static magnetizations in the spin valve have to be misaligned in order for the magnetization precession to produce a finite voltage. Because highly symmetric magnetization trajectories do not produce any variation of resistance with time in some cases, a third magnetic layer playing the role of an analyzer can be introduced. ${ }^{33}$ In ST-FMR, the noncollinearity of the magnetizations is also required for the $\mathrm{rf}$ spin transfer excitation not to vanish. ${ }^{25,26}$ Moreover, the latter was never directly compared to standard FMR, where a uniform rf magnetic field is used to excite SW modes. Thus, although the voltage detection of SW eigenmodes in hybrid nanostructures is elegant, one should keep in mind that some of them might be hidden due to symmetry reasons. 
Here we propose an independent method of detecting the magnetic resonance inside a spin-valve nanostructure. We use a magnetic resonance force microscope (MRFM). ${ }^{34-38}$ A first decisive advantage of the MRFM technique is that the detection scheme does not rely on the SW spatial symmetry because it measures the change in the longitudinal component of the magnetization. Like a bolometric detection, mechanical based FMR detects all the excited SW modes, independently of their phase. ${ }^{39,40}$ A second decisive advantage is that MRFM is a very sensitive technique that can measure the magnetization dynamics in nanostructures buried under metallic electrodes. ${ }^{41-43}$ Indeed, the probe is a magnetic particle attached at the end of a soft cantilever and is coupled to the sample through the dipolar interaction.

In our road map to characterize the nature of the autooscillation modes in STNOs, we report in this work on a comprehensive identification of the SW eigenmodes in the simplest possible geometry: the normally magnetized circular spin-valve nanopillar. This configuration is obtained by saturating the device with a large external magnetic field oriented perpendicular to the layers. Thanks to the preserved axial symmetry, a simplified spectroscopic signature of the different SW eigenmodes is expected. This identification is achieved experimentally from a comparative spectroscopic study of the SW eigenmodes excited either by an rf current flowing perpendicularly through the nanopillar, as used in STFMR, or by a homogeneous rf in-plane magnetic field, as used in conventional FMR. The paper shall be developed as follows. In Sec. II, we present the MRFM setup and the experimental protocol used to perform SW spectroscopy in a spin valve. We show that the SW spectrum excited by a homogeneous rf magnetic field is distinct from the SW spectrum excited by an rf current flowing through the nanopillar. In Sec. III, we perform unambiguous assignment of the resonance peaks to the different layers by experimental means. We determine which layer contributes mostly to each observed resonant signals by adding a direct current through the nanopillar, which produces opposite spin-transfer torques on each magnetic layer. In Sec. IV, we analyze the spectra by theoretical means using both a two-dimensional analytical formalism and a three-dimensional micromagnetic simulation package, SpinFlow 3D. By careful comparison of the measured spectra to the calculations, the nature of the SW dynamics in the system is identified and the selection rules for SW spectroscopy in perpendicularly magnetized spin-valve nanostructures are established. This result is completed in Sec. V by a study of the influence of symmetry breaking on the selection rules. This is obtained experimentally by introducing a tilt angle of the applied magnetic field and in simulations by changing the shape of the nanopillar. In the Conclusion, we emphasize the importance of this work for phase synchronization of STNOs. The paper is arranged in such a fashion so as to present the main results in the body of the text. Comprehensive appendixes have been put at the end of the paper, where the details of the introduced material are developed.

\section{FERROMAGNETIC RESONANCE FORCE SPECTROSCOPY}

This section starts with a description of the nanopillar sample, followed by a description of the MRFM instrument used for this spectroscopic study. Then, we compare the experimental SW spectra excited by an rf current flowing perpendicularly through the nanopillar, as used in ST-FMR, and by a uniform rf magnetic field applied parallel to the layers, as used in standard FMR.

\section{A. The lithographically patterned nanostructure}

The spin-valve structure used in this study is a standard permalloy $\left(\mathrm{Ni}_{80} \mathrm{Fe}_{20}=\mathrm{Py}\right)$ bilayer structure sandwiching a 10-nm copper $(\mathrm{Cu})$ spacer: The thicknesses of the thin $\mathrm{Py}_{a}$ and the thick $\mathrm{Py}_{b}$ layers are, respectively, $t_{a}=4 \mathrm{~nm}$ and $t_{b}=15 \mathrm{~nm}$. Special care has been put into the design of the microwave circuit around the nanopillar. The inset of Fig. 1 shows a scanning electron microscopy top view of this circuit. The nanopillar is located at the center of the cross-hair, in the middle of a highly symmetric pattern designed to minimize cross-talk effects between both rf circuits shown in blue and red, which provide two independent excitation means.

The nanopillar is patterned by standard e-beam lithography and ion-milling techniques from the extended film, $\left(\mathrm{Cu} 60\left|\mathrm{Py}_{b} 15\right| \mathrm{Cu} 10\left|\mathrm{Py}_{a} 4\right| \mathrm{Au} 25\right)$ with thicknesses expressed in $\mathrm{nm}$, to a nanopillar of nominal radius $100 \mathrm{~nm}$. A precise control makes it possible to stop the etching process exactly at the bottom $\mathrm{Cu}$ layer, which is subsequently used as the bottom contact electrode. A planarization process of a polymerized resist by reactive ion etching makes it possible to uncover the top of the nanopillar and to establish the top contact electrode. The top and bottom contact electrodes are shown in red tone in Fig. 1. These pads are impedance matched to allow for high-frequency characterization by injecting an $\mathrm{rf}$ current $i_{\text {rf }}$ through the device. The bottom $\mathrm{Cu}$ electrode is grounded and the top $\mathrm{Au}$ electrode is wire bounded to the

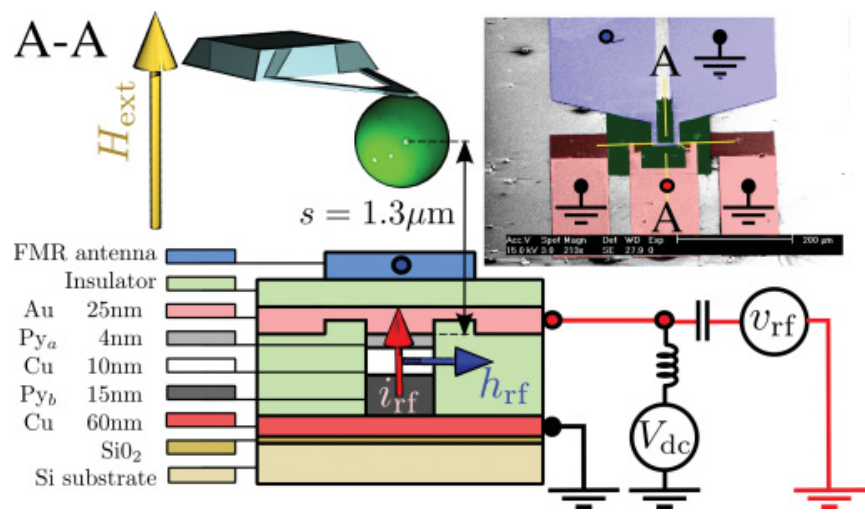

FIG. 1. (Color online) Schematic representation of the experimental setup used for this comparative SW spectroscopic study. The magnetic sample is a circular nanopillar comprising a thin $\mathrm{Py}_{a}$ layer and a thick $\mathrm{Py}_{b}$ magnetic layer separated by a $\mathrm{Cu}$ spacer. It is saturated by a large magnetic field $\boldsymbol{H}_{\text {ext }}$ applied along its normal axis. A cantilever with a magnetic sphere attached at its tip monitors the magnetization dynamics inside the buried structure. The inset is a microscopy image (top view) of the two independent excitation circuits: In red is the circuit allowing the injection of an rf current perpendicular to plane through the nanopillar $\left(i_{\mathrm{rf}}\right.$, red arrow); in blue is the circuit allowing the generation of an $\mathrm{rf}$ in-plane magnetic field $\left(h_{\mathrm{rf}}\right.$, blue arrow). The nanopillar is at the center of the yellow cross-hair. The main figure is a section along the $A-A$ direction. 
central pin of a microwave cable. Hereafter, spectra associated with SW excitations by this part of the microwave circuit are displayed in red tone. The nano pillar is also connected through a bias- $T$ to a dc current source and to a voltmeter through the same contact electrodes, which can be used for standard current-perpendicular-to-the-plane (CPP-GMR) transport measurements. ${ }^{44}$ In our circuit, a positive current corresponds to a flow of electrons from the $\mathrm{Py}_{b}$ thick layer to the $\mathrm{Py}_{a}$ thin layer and stabilizes the parallel configuration due to the spin-transfer effect. ${ }^{6,7}$ The studies presented below are limited to a dc current up to the threshold current for auto-oscillations in the thin layer.

The originality of our design is the addition of an independent top microwave antenna, whose purpose is to produce an in-plane rf magnetic field $h_{\mathrm{rf}}$ at the nanopillar location. In Fig. 1 this part of the microwave circuit is shown in blue. The broadband strip-line antenna consists of a 300-nm-thick $\mathrm{Au}$ layer evaporated on top of a polymer layer that provides electrical isolation from the rest of the structure. The width of the antenna constriction situated above the nanopillar is $10 \mu \mathrm{m}$. Injecting a microwave current from a synthesizer inside the top antenna produces a homogeneous in-plane linearly polarized microwave magnetic field oriented perpendicular to the stripe direction. Hereafter, spectra associated with SW excitations by this part of the microwave circuit are displayed in blue tone.

\section{B. Mechanical FMR}

The nanofabricated sample is then mounted inside a MRFM, hereafter named mechanical FMR. ${ }^{38}$ The whole apparatus is placed inside a vacuum chamber $\left(10^{-6}\right.$ mbar $)$ operated at room temperature. The external magnetic field produced by an electromagnet is oriented out of plane, that is, along the nanopillar axis $\hat{z}$. The mechanical-FMR setup allows for a precise control, within $0.2^{\circ}$, of the polar angle between the applied field and $\hat{z}$. In our study, the strength of the applied magnetic field shall exceed the saturation field $(\approx 8 \mathrm{kOe})$, so that the nanopillar is studied in the saturated regime.

The mechanical detector is an ultrasoft cantilever, an Olympus Bio-Lever having a spring constant $k \approx 5 \mathrm{mN} / \mathrm{m}$, with a 800-nm-diameter sphere of soft amorphous Fe (with 3\% $\mathrm{Si}$ ) glued to its apex. Standard piezo displacement techniques allow for positioning the magnetic spherical probe precisely above the center of the nanopillar, so as to retain the axial symmetry. This is obtained when the dipolar interaction between the sample and the probe is maximal, by minimizing the cantilever resonance frequency, which is continuously monitored. ${ }^{41}$

The mechanical sensor is insensitive to the rapid oscillations of the transverse component in the sample, which occur at the Larmor precession frequency, that is, several orders of magnitude faster than its mechanical resonances. The dipolar force on the cantilever probe is thus proportional to the static component of the magnetization inside the sample. For our normally magnetized sample, this longitudinal component reduces to $M_{z}$. We emphasize that for a bilayer system, the force signal integrates the contribution of both layers. Moreover, the local $M_{z}(\boldsymbol{r})$ in the two magnetic layers is weighted by the distance dependence of the dipolar coupling to the center of the sphere. In our case though, where the separation between the sphere and the sample is much larger than the sample dimensions, one can neglect this weighting and the measured quantity simplifies to the spatial average:

$$
\left\langle M_{z}\right\rangle \equiv \frac{1}{V} \int_{V} M_{z}(\boldsymbol{r}) d^{3} \boldsymbol{r},
$$

where the chevron brackets stand for the spatial average over the volume of the magnetic body.

The mechanical-FMR spectroscopy presented below consists of recording by optical means the vibration amplitude of the cantilever either as a function of the out-of-plane magnetic field $H_{\text {ext }}$ at a fixed microwave excitation frequency $f_{\text {fix }}$ or as a function of the excitation frequency $f$ at a fixed magnetic field $H_{\text {fix }}$. This type of spectroscopy is called cw, for continuous wave, as it is monitoring the magnetization dynamics in the sample under a forced regime. A source modulation is applied on the cw excitation. It consists of a cyclic absorption sequence, where the microwave power is switched on and off at the cantilever resonance frequency, $f_{c} \approx$ $11.85 \mathrm{kHz}$. The signal is thus proportional to $\left\langle\Delta M_{z}\right\rangle$, where $\Delta$ represents the difference from the thermal equilibrium state. The source modulation enhances the signal, recorded by a lock-in detection, by the quality factor $Q \approx 2000$ of the mechanical oscillator. The force sensitivity of our mechanicalFMR setup is better than $1 \mathrm{fN}$, corresponding to less than $10^{3}$ Bohr magnetons in a bandwidth of $1 \mathrm{~s}$ (Ref. 38). We note that this modulation technique does not affect the line shape in the linear regime, because the period of modulation $1 / f_{c}$ is very large compared to the relaxation times of the studied ferromagnetic system. ${ }^{46,47}$ Moreover, we emphasize that since the mechanical-FMR signal originates from the cyclic diminution of the spatially averaged magnetization inside the whole nanopillar synchronous with the absorption of the microwave power, it detects all possible SW modes without discrimination. ${ }^{39,40}$

Finally, we mention that the stray field produced by the magnetic sphere attached on the cantilever does affect the detected SW spectra. In our setup, the separation between the center of the spherical probe and the nanopillar is set to $1.3 \mu \mathrm{m}$ (see Fig. 1), which is a large distance considering the lateral size of the sample. At such distance, the coupling between the sample and the probe is weak $^{38}$ as it does not affect the profiles of the intrinsic SW modes in the sample. This is in contrast with the strong coupling regime, where the stray field of the magnetic probe can be used to localize SW modes below the MRFM tip. ${ }^{48}$ For our mechanical SW spectrometer, the perturbation of the magnetic sphere reduces to a uniform translation of all the peak positions ${ }^{49}$ by -190 Oe (see Sec. III B). In the following, all the SW spectra are recorded with the magnetic sphere at the same exact position above the nanopillar.

\section{C. rf magnetic field vs rf current excitations}

The comparative spectroscopic study performed by mechanical FMR at $f_{\text {fix }}=8.1 \mathrm{GHz}$ on the normally magnetized spin-valve nanopillar is presented in Fig. 2. In these experiments, there is no dc current flowing through the device, and the spectra are obtained in the small excitation regime 

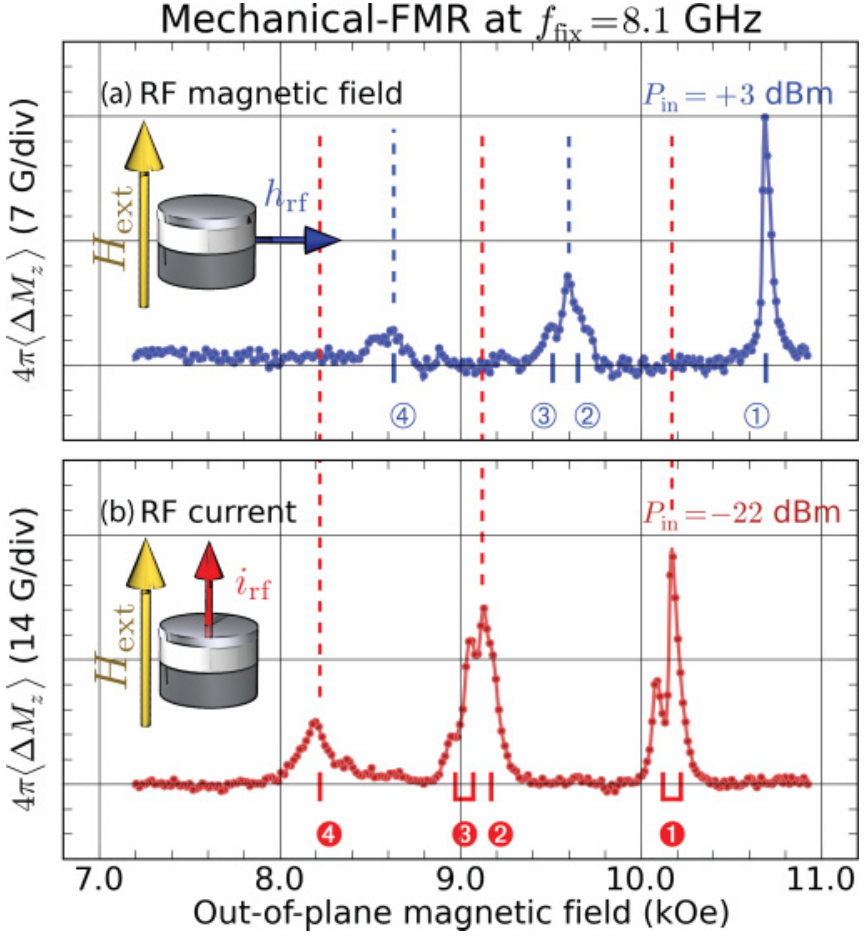

FIG. 2. (Color online) Comparative spectroscopic study performed by mechanical FMR at $f_{\text {fix }}=8.1 \mathrm{GHz}$, demonstrating that distinct SW spectra are excited by a uniform in-plane rf magnetic field (a) and by an rf current flowing perpendicularly through the layers (b). The positions of the peaks are reported in Table II.

(precession angles less than $5^{\circ}$; see Appendix B 1). The top panel (a) shows the SW spectrum excited by a uniform rf magnetic field applied in the plane of the layers, while the bottom panel (b) displays the SW spectrum excited by an rfF current flowing perpendicularly through the magnetic layers. The striking result is that these two spectra are different: None of the SW modes excited by the homogeneous rf field is present in the spectrum excited by the rf current flowing through the nanopillar, and vice versa.

Let us first focus on Fig. 2(a), where the obtained absorption spectrum corresponds to the so-called standard FMR spectrum. Here, the output power of the microwave synthesizer at 8.1 $\mathrm{GHz}$ is set to $+3 \mathrm{dBm}$, which corresponds to an amplitude of the uniform linearly polarized rf magnetic field $h_{\mathrm{rf}} \simeq 2.1 \mathrm{Oe}$ produced by the antenna (see Appendix B 1). In this standard FMR spectrum, only SW modes with nonvanishing spatial average can couple to the homogeneous rf field excitation. In field-sweep spectroscopy, the lowest energy mode occurs at the largest magnetic field. So, the highest field peak at $H_{(1)}=$ $10.69 \mathrm{kOe}$ should be ascribed to the uniform mode. Since this peak is also the largest of the spectrum, it corresponds to the precession of a large volume in the nanopillar; that is, the thick layer must dominate in the dynamics. In mechanical FMR, a quantitative measurement of the longitudinal magnetization is obtained $^{39,50}$ (see Appendix B 1). The amplitude of the peak at $H_{(1)}$ corresponds to $4 \pi\left\langle\Delta M_{z}\right\rangle \simeq 14 \mathrm{G}$, which represents a precession angle $\langle\theta\rangle \simeq 3.1^{\circ}$. This sharp peak is followed by a broader peak with at least two maxima at $H_{(2)}=9.65 \mathrm{kOe}$ and $H_{3}=9.51 \mathrm{kOe}$, and at lower field, by a smaller resonance around $H_{(4}=8.64 \mathrm{kOe}$. Among these other peaks, there is the uniform mode dominated by the thin layer, which has to be identified and distinguished from higher radial index SW modes.

Let us now turn to Fig. 2(b), corresponding to the spectroscopic response to an $\mathrm{rf}$ current of same frequency $8.1 \mathrm{GHz}$ flowing perpendicularly through the nanopillar. Here, the output power of the microwave synthesizer is $-22 \mathrm{dBm}$, which corresponds to an rms amplitude of the rf current $i_{\mathrm{rf}} \simeq 170 \mu \mathrm{A}$ (see Appendix B 2). The SW spectrum is acquired under the exact same conditions as for standard FMR; that is, the spherical magnetic probe of the mechanical-FMR detection is kept at the same location above the sample. The striking result is that the positions of the peaks in Figs. 2(a) and 2(b) do not coincide. More precisely, there seems to be a translational correspondence between the two spectra, which are shifted in field by about $0.5 \mathrm{kOe}$ from each other. The lowest energy mode in the rf current spectrum occurs at $H_{\mathbf{0}}=10.22 \mathrm{kOe}$. This is again the most intense peak, suggesting that the thick layer contributes to it, and $4 \pi\left\langle\Delta M_{z}\right\rangle \simeq 26 \mathrm{G}$, which represents a precession angle $\langle\theta\rangle \simeq 4.2^{\circ}$. This main resonance line is also split in two peaks, with a smaller resonance in the low field wing of the main peak, about 100 Oe away. At lower field, two distinct peaks appear at $H_{\mathbf{B}}=9.17 \mathrm{kOe}$ and $H_{\mathbf{B}}=9.07 \mathrm{kOe}$ and another peak is visible at $H_{\boldsymbol{\Phi}}=8.22 \mathrm{kOe}$.

The fact that the two spectra of Figs. 2(a) and 2(b) are distinct implies that they have a different origin. It will be shown in the theoretical Sec. IV A 3 that the rf field and the rf current excitations probe two different azimuthal symmetries $\ell$. Namely, only $\ell=0$ modes are excited by the uniform $\mathrm{rf}$ magnetic field, whereas only $\ell=+1$ modes are excited by the orthoradial rf Oersted field associated with the rf current. ${ }^{51}$ The mutually exclusive nature of the responses to the uniform and orthoradial symmetry excitations is a property of the preserved axial symmetry, where the azimuthal index $\ell$ is a good quantum number; that is, different $\ell$-index modes are not mixed and can be excited separately (see Sec. IV A 2).

\section{EXPERIMENTAL ANALYSIS}

In this section, we first look at the effect of a continuous current flowing through the nanopillar on the SW spectra in order to determine which layer contributes most to the resonant signals observed in Fig. 2. Due to the asymmetry of the spin transfer torque in each magnetic layer, the different SW modes are influenced differently depending on the layer in which the precession is the largest. Then we briefly mention experiments where spectroscopy is performed by monitoring the dc voltage produced by the magnetization precession in the hybrid nanostructure and compared to mechanical FMR. Finally, the analysis of the frequency-field dispersion relation and of the linewidth of the resonance peaks makes it possible to extract the gyromagnetic ratio and the damping parameters in the thick and thin layers.

\section{A. Direct bias current}

To gain further insight about the peak indexation, we have measured the spectral evolution produced on the SW spectra of Fig. 2 when a finite dc current $I_{\mathrm{dc}} \neq 0$ is injected in the 

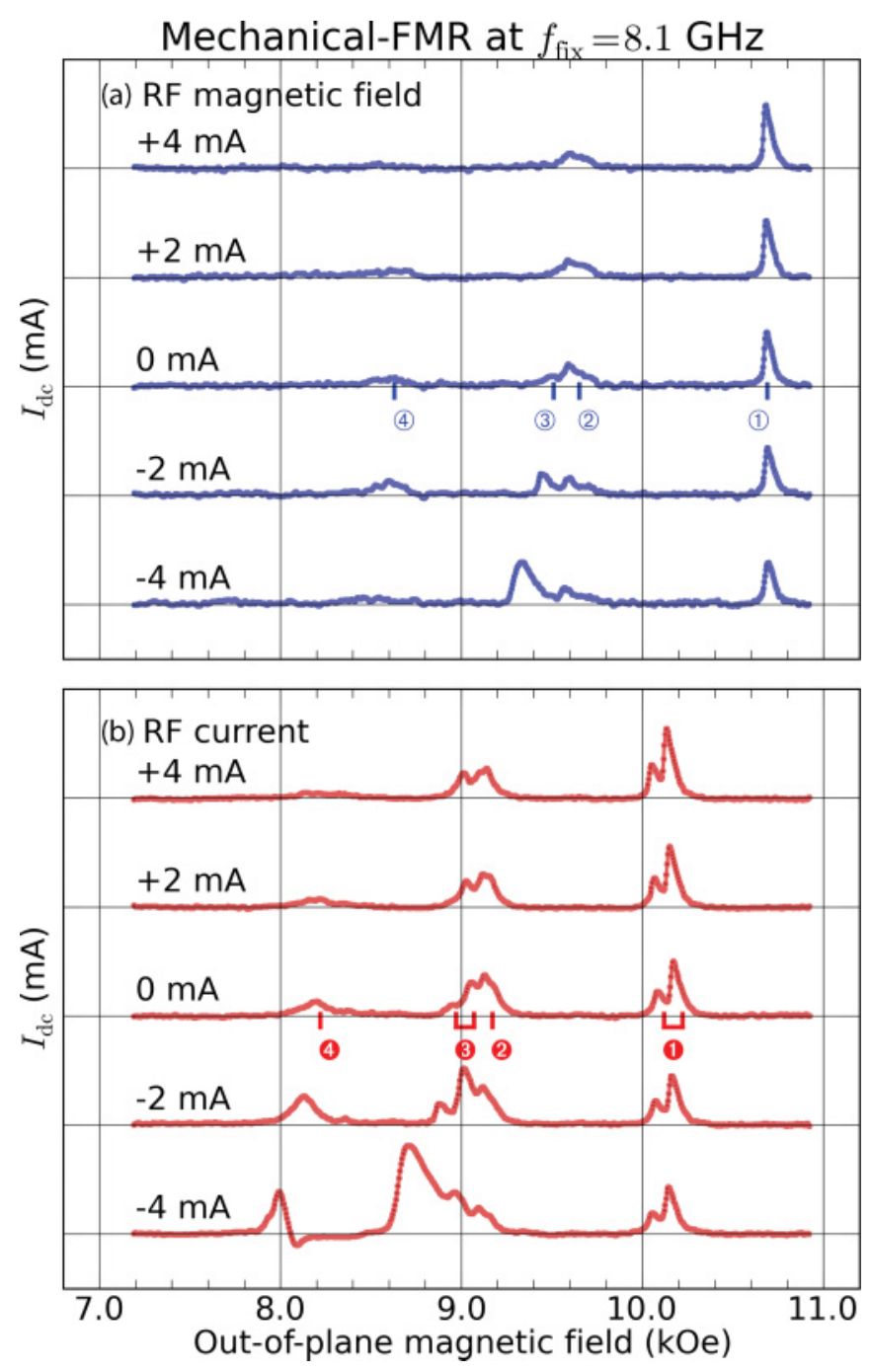

FIG. 3. (Color online) Evolution of the SW spectra measured at $f_{\text {fix }}=8.1 \mathrm{GHz}$ by mechanical FMR for different values of the continuous current $I_{\mathrm{dc}}$ flowing through the nanopillar. Panel (a) corresponds to excitation by a uniform rf magnetic field, and panel (b) to excitation by an rf current through the sample.

nanopillar. We recall that for our sign convention, a positive $\mathrm{dc}$ current stabilizes the thin layer and destabilizes the thick one due to the spin transfer torque, and vice versa. ${ }^{6,7}$ The results obtained by mechanical-FMR are reported in Fig. 3 .

Let us first concentrate on Fig. 3(a), in which the excitation that probes the different SW modes is the same as in Fig. 2(a), that is, a uniform rf magnetic field. Two main features can be observed in the evolution of the SW spectra as $I_{\mathrm{dc}}$ is varied. First, the amplitude of the peak at $H_{(1}$ smoothly increases with the positive current and smoothly decreases with the negative current. At the same time, the peak at $H_{\overparen{3}}$, which is about five times smaller than the peak at $H_{(1}$ when $I_{\mathrm{dc}}=0 \mathrm{~mA}$, almost disappears for positive current and strongly increases at negative current, until it becomes larger than the other peaks when $I_{\mathrm{dc}}=-4 \mathrm{~mA}$. These two features are consistent with the effect of spin transfer if we ascribe the peak at $H_{(1)}$ to the uniform mode of mostly the thick layer and the peak at $H_{3}$ to the one of mostly the thin layer. More precisely, it is expected that in the subcritical regime $\left(\left|I_{\mathrm{dc}}\right|<\right.$ $I_{\text {th }}$, where $I_{\text {th }}$ is the threshold current for auto-oscillations, $I_{\text {th }}<0$ for the thin layer and $I_{\text {th }}>0$ for the thick layer), the damping scales as $\alpha\left(1-I_{\mathrm{dc}} / I_{\mathrm{th}}\right)^{25,26}$ (see Appendix A 1 ), where $\alpha$ is the Gilbert damping parameter. It means that the linewidth of a resonance peak that is favored by spin transfer should decrease as the current gets closer to $I_{\text {th }}$, and that its amplitude, which scales as the inverse linewidth, should increase.

Although the effect on the peak amplitude noted above is clear in Fig. 3(a), it is not on the linewidth. The reason is that in this experiment, the strength of the driving rf magnetic field is kept constant to $h_{\mathrm{rf}}=2.1 \mathrm{Oe}$. As a result, the shape of the growing peaks in Fig. 3(a) becomes more asymmetric, which is a signature that the precession amplitude driven by the $\mathrm{rf}$ field is strong enough to change the internal field by an amount of the order of the linewidth. This leads to some foldover of the resonance line, ${ }^{52,53}$ a nonlinear effect for which details are given in the Appendix B 1. In other words, the distortion of the line shape as the peak amplitude increases prevents seeing the diminution of its linewidth. ${ }^{54}$ It would be necessary to decrease the excitation amplitude as the threshold current is approached $^{26}$ so as to maintain the peak amplitude in the linear regime in order to reveal it.

The opposite signs of the spin-transfer torques which influence the dynamics in the thin and thick layers are thus clearly seen in Fig. 3(a). Their relative strengths can also be determined, as the amplitude of the peak at $H_{3}$ grows much faster with negative current than the one of the peak at $H_{(1}$ with positive current. This is because the efficiency of the spin transfer torque is inversely proportional to the thickness of the layer. ${ }^{6,7}$ Whereas the precession angle in the thick layer does not vary much with $I_{\mathrm{dc}}$ (from $\approx 2.5^{\circ}$ at $-4 \mathrm{~mA}$ to $\approx 3.5^{\circ}$ at +4 $\mathrm{mA}$ ), the precession angle that can be deduced from $\left\langle\Delta M_{z}\right\rangle$ in the thin layer grows from almost zero at $I_{\mathrm{dc}}=+4 \mathrm{~mA}$ to more than $6^{\circ}$ at $I_{\mathrm{dc}}=-4 \mathrm{~mA}$. Moreover, the peak position $H_{3}$ shifts clearly toward lower field as the negative current is increased. This is due to the onset of spin-transfer-driven auto-oscillations in the thin layer, which occurs at a threshold current $I_{\text {th }} \lesssim-4 \mathrm{~mA}$ and produces this nonlinear shift. ${ }^{19}$ We note that such a value for the threshold current in the thin layer can be found from Slonczewski's model (see Appendix A 1).

Let us now briefly discuss Fig. 3(b), which shows the dependence on $I_{\mathrm{dc}}$ of the mechanical-FMR spectra excited by an rf current excitation. A similar dependence on $I_{\mathrm{dc}}$ of the resonance peaks in translational correspondence with Fig. 3(a) is observed. Again, a clear asymmetry is revealed depending on the polarity of $I_{\mathrm{dc}}$ and on the SW modes. The double peak at $H_{\mathbf{0}}$ is favored by positive currents; hence, it should be ascribed to mostly the thick-layer precessing. The double peak at $H_{\boldsymbol{B}}$ is strongly favored by negative currents; hence, it should be ascribed to mostly the thin-layer precessing. Moreover, a careful inspection shows that the peak $H_{\boldsymbol{Q}}$, which looks single at $I_{\mathrm{dc}}=0 \mathrm{~mA}$, is actually at least double. We explain this splitting of higher harmonics modes in Sec. V B.

To summarize, the passage of a dc current through the nanopillar makes it possible to determine which layer mostly contributes to the observed SW modes, owing to the asymmetry of the spin transfer effect. 
TABLE I. Magnetic parameters of the thin $\mathrm{Py}_{a}$ and thick $\mathrm{Py}_{b}$ layers measured by cavity FMR on the reference film (top row) and by mechanical FMR in the nanopillar (bottom row).

\begin{tabular}{lcccc}
\hline \hline $4 \pi M_{a}(\mathrm{G})$ & $\alpha_{a}$ & $4 \pi M_{b}(\mathrm{G})$ & $\alpha_{b}$ & $\gamma\left(\mathrm{rad} \mathrm{s}^{-1} \mathrm{G}^{-1}\right)$ \\
\hline $8.2 \times 10^{3}$ & $1.5 \times 10^{-2}$ & $9.6 \times 10^{3}$ & $0.9 \times 10^{-2}$ & $1.87 \times 10^{7}$ \\
$8.0 \times 10^{3}$ & $1.4 \times 10^{-2}$ & $9.6 \times 10^{3}$ & $0.85 \times 10^{-2}$ & $1.87 \times 10^{7}$ \\
\hline \hline
\end{tabular}

\section{B. Voltage FMR}

Our experimental setup also makes it possible to monitor the dc voltage produced across the nanopillar by the precession of the magnetization in the bilayer structure. A lock-in detection is used to measure the difference of voltage across the nanopillar when the rf is on and off: $V_{\mathrm{dc}}=V_{\mathrm{on}}-V_{\mathrm{off}}$. This can be done simultaneously to the acquisition of the mechanical-FMR signal, under the exact same conditions (see Fig. 1). Since the presentation of the experimental results requires a specific discussion, the details as well as the graphs will be published elsewhere. Here we only reveal the three main features that can be noticed in the voltage-FMR spectra.

First, even at $I_{\mathrm{dc}}=0$, dc voltage peaks are produced across the nanopillar at the same positions as the mechanical-FMR peaks observed in Fig. 2, with a difference of potential that lies in the $10-\mathrm{nV}$ range for the precession angles excited here. It is ascribed to spin pumping and accumulation in the spin-valve hybrid structure. ${ }^{55,56}$ Second, these voltage resonance peaks are signed; namely, the $\mathrm{SW}$ modes favored at $I_{\mathrm{dc}}<0$ in Fig. 3(a) (for which the thin layer is dominating) produce a positive voltage peak, whereas those favored at $I_{\mathrm{dc}}>0$ (thick layer dominating) produce a negative voltage peak. This difference between the thick- and thin-layer contributions is ascribed to the asymmetry of the spin accumulation in the multilayer stack. ${ }^{57}$ Third, the relative amplitudes of the voltage-FMR peaks are different from the mechanical-FMR ones. For instance, the voltage-FMR peak of the thin layer at $H_{(3}$ is slightly larger than the peak at $H_{(1)}$ of the thick layer (and it has an opposite sign). This illustrates an important difference between the two detection schemes. While mechanical-FMR measures a quantity proportional to the precessing volume, $\left\langle\Delta M_{z}\right\rangle$, the voltage-FMR measures an interfacial effect. Therefore, when the same precession angle is excited in both layers, the voltage-FMR signal associated with each layer is approximately the same, whereas the mechanical-FMR signal from the thin layer is roughly four times smaller than the one from the thick layer, due to their relative thicknesses.

Finally, we mention that voltage-FMR spectroscopy can also record the intrinsic FMR spectrum of the nanopillar, that is, in the absence of the spherical MRFM probe above it. This makes it possible to check that the only effect introduced by the probe in mechanical-FMR is an overall shift of the SW modes spectra to lower field without any other distortion, and to quantify this shift, found to be -190 Oe (Ref. 58).

\section{Gyromagnetic ratio}

A precise orientation of the applied magnetic field $\boldsymbol{H}_{\text {ext }}$ along the normal $\hat{z}$ of the sample [polar angle $\theta_{H}=\left(\hat{z}, \boldsymbol{H}_{\text {ext }}\right)=$ 0] enables a direct determination of the modulus $\gamma$ of the gyromagnetic ratio. ${ }^{38}$ By following the frequency-field dispersion relation of the resonance peaks at $H_{(1)}$ and at $H_{(3}$ (from 4.5 to $8.1 \mathrm{GHz}$ and from 6.2 to $11 \mathrm{GHz}$, respectively) in our nanopillar, it is found that $\gamma=1.87 \times 10^{7} \mathrm{rad} \mathrm{s}^{-1} \mathrm{G}^{-1}$ is identical in the thick and thin layers. Moreover, the value of $\gamma$ measured in the nanopillar is the same as in the extended reference film (see Appendix B 3 and Table I), confirming that the applied field is sufficient to saturate the two magnetic layers and is precisely oriented along $\hat{z}$.

The same result is obtained by following the evolution of the frequency-field dispersion relation presented in Fig. 4. Here we take advantage of the broadband design of the electrodes which connect the nanopillar to measure the FMR spectrum at fixedbias magnetic field, $H_{\text {fix }}=10 \mathrm{kOe}$, by sweeping the frequency of the rf current through it. The data are plotted according to the frequency scale above Fig. 4(a). At constant magnetic configuration (above the saturation field, that is, $\gtrsim 8 \mathrm{kOe}$ ), this frequency scale is in correspondence with field-sweep experiments performed at fixed rf frequency $f_{\text {fix }}=8.1 \mathrm{GHz}$ through
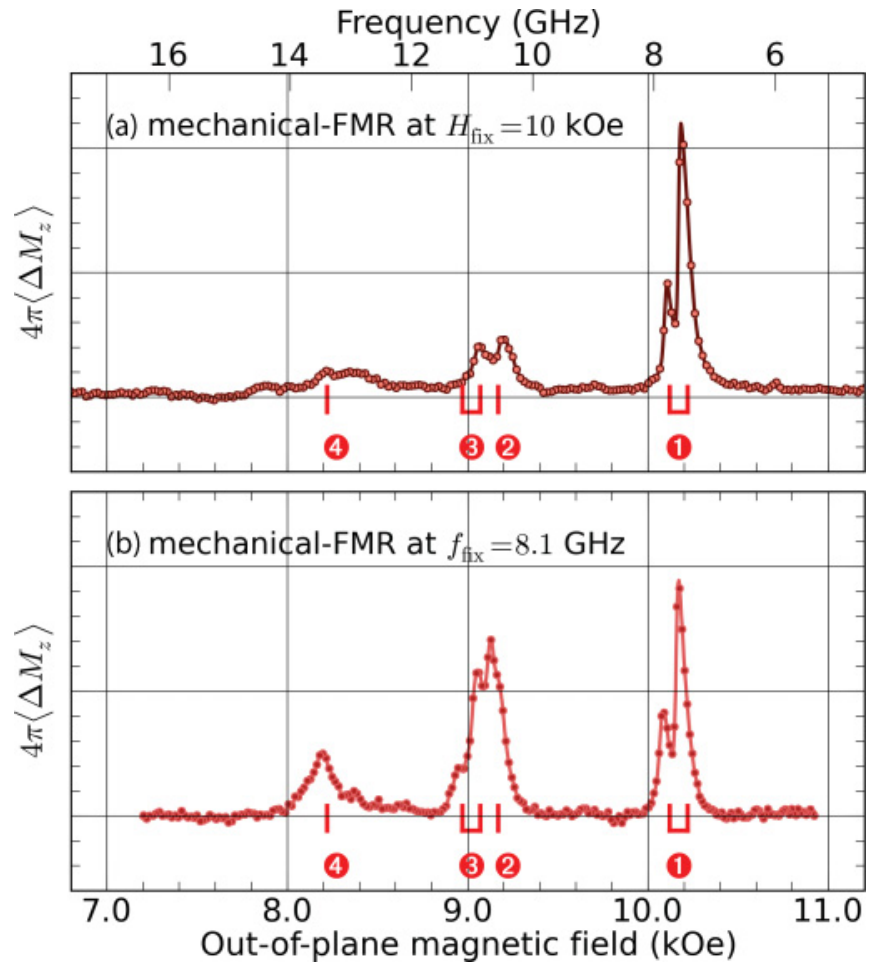

FIG. 4. (Color online) Frequency-field dispersion relation: the top spectrum (a) is measured at fixed-bias field $H_{\text {fix }}=10 \mathrm{kOe}$ by sweeping the frequency, $f$, of the $\operatorname{rf}$ current $i_{\mathrm{rf}}$ through the nanopillar. The bottom spectrum (b) is the same as in Fig. 2(b), and is obtained by sweeping the external magnetic field, $H_{\text {ext }}$, at fixed frequency $f_{\text {fix }}=8.1 \mathrm{GHz}$ of $i_{\mathrm{rf}}$. The top and bottom scales are in correspondence through the affine transformation $H_{\mathrm{ext}}-H_{\text {fix }}=2 \pi\left(f-f_{\text {fix }}\right) / \gamma$. 
the affine transformation $H_{\text {ext }}-H_{\text {fix }}=2 \pi\left(f-f_{\text {fix }}\right) / \gamma$, as seen from the field scale below Fig. 4(b). This is a direct experimental check of the equivalence between frequency and field sweep experiments in the normally saturated state.

\section{Damping parameters}

From the FMR data presented above, we can also directly extract the damping parameters in each permalloy layer. Indeed, in field-sweep spectroscopy in the normal orientation $\left(\theta_{H}=0\right)$, the full width at half maximum (FWHM) $\Delta H$ of a resonance line is proportional to the excitation frequency $\omega /(2 \pi)$ through the Gilbert constant $\alpha: \Delta H=2 \alpha(\omega / \gamma)$ (see Appendix A 1).

The linewidth of the peak at $H_{(1}$ associated with mainly the thick layer in Fig. 2(a) is equal to $\Delta H_{(1}=48$ Oe, which corresponds to a damping $\alpha_{(1)}=0.88 \times 10^{-2}$. From the same mechanical-FMR spectrum, the linewidth of the peak at $H_{3}$, associated with mainly the thin layer, cannot be easily extracted due to the proximity of the peak at $H_{(2)}$. Owing to the interfacial origin of the voltage-FMR signal, the peak at $H_{3}$ is more distinguishable in the spectrum of the voltage FMR (not shown), and its linewidth, $\Delta H_{3}=70 \mathrm{Oe}$, can be fitted. It corresponds to a damping $\alpha_{3}=1.29 \times 10^{-2}$.

The linewidths of the modes at $H_{\mathbf{0}}$ and $H_{\boldsymbol{B}}$ can also be fitted and give similar results for the damping associated with each layer. In the case of the rf current excitation, a frequency-sweep spectrum can be acquired at a fixed-bias magnetic field $H_{\text {fix }}$ (see Fig. 4). In that case, the damping constant is simply obtained by $\alpha=\Delta f /(2 f)$, where $\Delta f$ is the width of the line centered at $f$. At $H_{\text {fix }}=10 \mathrm{kOe}, f_{\mathbf{o}}=7.37$ $\mathrm{GHz}$ and $\Delta f_{\mathbf{0}}=0.12 \mathrm{GHz}$, which yield $\alpha_{\mathbf{0}}=0.81 \times 10^{-2}$, and $f_{\boldsymbol{B}}=10.92 \mathrm{GHz}$ and $\Delta f_{\boldsymbol{\theta}}=0.33 \mathrm{GHz}$, which yield $\alpha_{\text {B }}=1.5 \times 10^{-2}$.

In summary, we retain the following values for the damping parameters in the thin and the thick layers, respectively: $\alpha_{a}=$ $(1.4 \pm 0.2) \times 10^{-2}$ and $\alpha_{b}=(0.85 \pm 0.1) \times 10^{-2}$. We have reported them, together with $\gamma$, in Table I.

These two values are in line with the ones obtained on the reference film, which have also been reported in Table I. Still, we observe that the linewidths in the nanostructure are systematically lower than the ones measured on the reference film. This is a constant characteristic that we associate with the confined geometry, which lifts most of the degeneracy (well separated SW modes) and thus strongly reduces the inhomogeneous part of the linewidth observed in the infinite layer. ${ }^{15,26}$ Rather, the inhomogeneities associated with the magnetic layers ${ }^{15}$ or with the confinement geometry will lead to some mode splitting in the nanostructure (see Sec. V B). We have checked that the inhomogeneous contribution to the linewidth in the nanopillar is weak by following the dependence of the measured $\Delta H$ as a function of frequency. In fact, the increase of $\Delta H_{3}$ from $70 \mathrm{Oe}$ at $8.1 \mathrm{GHz}$ to $105 \mathrm{Oe}$ at $11 \mathrm{GHz}$ is purely homogeneous.

Finally, the finding that the damping is larger in the thin layer than in the thick layer is ascribed to the adjacent metallic layers. ${ }^{59}$ In fact, nonlocal effects such as the spin pumping effect $^{55,60}$ and the spin diffusion in the adjacent normal layers by the conduction electrons yield an interfacial increase of the magnetic damping ${ }^{61}$ that is stronger in the case of thin layers.

\section{THEORETICAL ANALYSIS}

In this section, we first review a general formalism allowing the calculation of the discrete spectrum associated with SW propagation inside a confined body of arbitrary magnetic configuration. It is shown that in the two-dimensional (2D) axially symmetric case, different $\ell$-index modes can be excited separately, as found experimentally in Sec. II C. The classification of the SW modes in this case is also used to extract the parameters of each magnetic layer from the experimental FMR spectra. In a second part, we discuss the influence of the dynamic coupling between the magnetic disks, where the collective dynamics splits into binding and antibinding modes. It is shown that in our experimental case, the dynamic dipolar coupling introduces a weak spectral shift, although its influence on the character of the SW modes is real. In the last part, a comparison to full three-dimensional (3D) micromagnetic simulations is performed in order to study in details the collective dynamics in the nanopillar.

\section{A. Analytical model}

\section{General theory}

Below, we briefly review the general theory of linear SW excitations (see Appendix A 1 for more details). We consider an arbitrary equilibrium magnetic configuration, where the local magnetization writes $M_{s} \hat{\boldsymbol{u}}$, with $M_{s}$ the saturation magnetization and $\hat{\boldsymbol{u}}$ the unit vector along the local equilibrium direction (implicitly dependent on the spatial coordinates). The linearization of the local equation of motion is obtained by decomposing the instantaneous magnetization vector $\boldsymbol{M}(t)$ into a static and dynamic component ${ }^{63}$ (see Fig. 5). We use the following ansatz:

$$
\frac{\boldsymbol{M}(t)}{M_{s}}=\hat{\boldsymbol{u}}+\boldsymbol{m}(t)+\mathcal{O}\left(\boldsymbol{m}^{2}\right),
$$

where the transverse component $\boldsymbol{m}(t)$ is the small dimensionless deviation $(|\boldsymbol{m}| \ll 1)$ of the magnetization from the equilibrium direction. In ferromagnets, $|\boldsymbol{M}|=M_{s}$ is a constant of the motion, so that the local orthogonality condition $\hat{\boldsymbol{u}} \cdot \boldsymbol{m}=$ 0 is required.

Substituting Eq. (2) in the lossless Landau-Lifshitz equation Eq. (A1) (see Appendix A 1) and keeping only the terms linear in $\boldsymbol{m}$, one obtains the following dynamical equation for $\boldsymbol{m}$ :

$$
\frac{\partial \boldsymbol{m}}{\partial t}=\hat{\boldsymbol{u}} \times \widehat{\boldsymbol{\Omega}} * \boldsymbol{m},
$$

where here and henceforth, tensor operators are indicated by a wide hat, the cross product is denoted by $\times$, and the convolution product is denoted by $*$. The self-adjoint tensor operator $\widehat{\boldsymbol{\Omega}}$ represents the Larmor frequency:

$$
\widehat{\boldsymbol{\Omega}}=\gamma H \widehat{\boldsymbol{I}}+4 \pi \gamma M_{s} \widehat{\boldsymbol{G}},
$$

where $\gamma$ is the modulus of the gyromagnetic ratio, $H$ is the scalar effective magnetic field, $\boldsymbol{I}$ is the identity matrix, and $\widehat{\boldsymbol{G}}$ is the linear tensor operator describing the magnetic selfinteractions. The later is the addition of several contributions $\widehat{\boldsymbol{G}}^{(d)}+\widehat{\boldsymbol{G}}^{(e)}+\cdots$, respectively the magnetodipolar interactions, the inhomogeneous exchange, etc. (see Appendix A 2). 


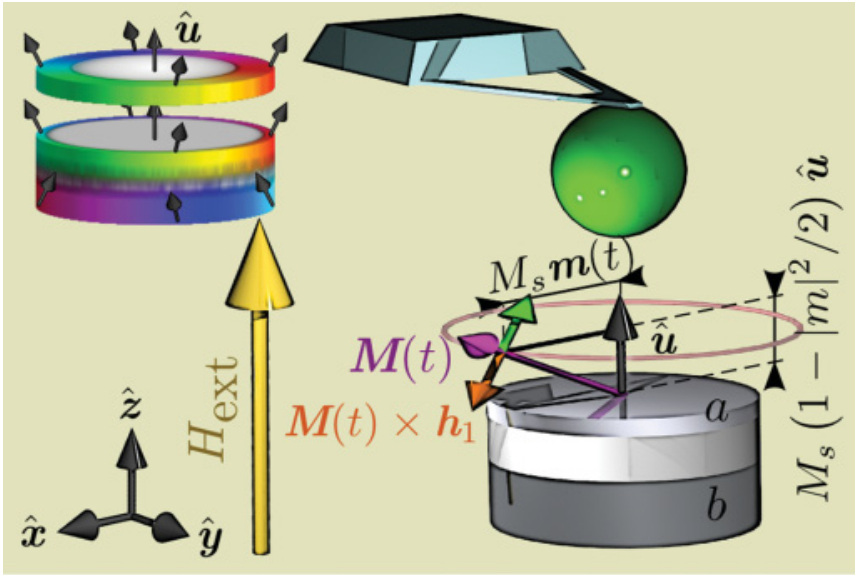

FIG. 5. (Color online) Schematic representation of the magnetization dynamics under continuous rf excitation. In the steady state, the torque exerted by the rf perturbation field $\boldsymbol{h}_{1}$ (orange arrow) compensates the torque exerted by the damping (green), and the local magnetization vector $\boldsymbol{M}(t)$ (purple) precesses at the Larmor frequency on a circular orbit ${ }^{62}$ around the local equilibrium direction (unit vector $\hat{\boldsymbol{u}}$ ). $\boldsymbol{M}(t)$ is the vector sum of a small oscillating component $M_{s} \boldsymbol{m}$ and a large static component $M_{s}\left(1-|\boldsymbol{m}|^{2} / 2\right)$, respectively, transverse and parallel to $\hat{\boldsymbol{u}}$. The inset shows the simulated spatial distribution of $\hat{\boldsymbol{u}}$ inside the nanopillar at $\boldsymbol{H}_{\mathrm{ext}}=$ $10 \mathrm{kOe}$ (see Sec. IV C). In the white regions, the magnetization is aligned along the normal $\hat{z}$ within $0.05^{\circ}$. In the colored regions, $\hat{\boldsymbol{u}}$ is flaring $\left(<5^{\circ}\right)$ in the radial direction (the hue indicates the direction of $\hat{\boldsymbol{u}}-\hat{z}$ according to the color code defined in Fig. 6).

The effective magnetic field $\boldsymbol{H}$ is a vector aligned along $\hat{\boldsymbol{u}}$, whose norm is

$$
H=\hat{\boldsymbol{u}} \cdot \boldsymbol{H}_{0}-4 \pi M_{s} \hat{\boldsymbol{u}} \cdot \widehat{\boldsymbol{G}} * \hat{\boldsymbol{u}},
$$

the sum of the $\hat{\boldsymbol{u}}$ component of $\boldsymbol{H}_{0}$, the total applied magnetic field including the stray field of any nearby magnetic object (in our case, the adjacent magnetic layer in the nanopillar and the spherical probe), reduced by the static self-interactions, which include the depolarization magnetic field along $\hat{\boldsymbol{u}}$ created by the static component of the magnetization.

SW modes $\boldsymbol{m}_{v}$ are by definition eigensolutions of Eq. (3):

$$
-i \omega_{v} \boldsymbol{m}_{v}=\hat{\boldsymbol{u}} \times \widehat{\boldsymbol{\Omega}} * \boldsymbol{m}_{v} .
$$

Here $\omega_{v}$ is the SW eigenfrequency and $v$ is a set of indices to enumerate the different modes.

The main properties of SW excitations follow from the eigenproblem Eq. (6) and the fact that the operator $\widehat{\boldsymbol{\Omega}}$ is selfadjoint and real. One can show that the eigensolutions obey the closure relation

$$
i\left\langle\overline{\boldsymbol{m}}_{v} \cdot\left(\hat{\boldsymbol{u}} \times \boldsymbol{m}_{v^{\prime}}\right)\right\rangle=\mathcal{N}_{v} \delta_{v, v^{\prime}},
$$

where $\delta$ is the Kronecker delta function and $\overline{\boldsymbol{m}}$ stands for the complex conjugate of $\boldsymbol{m}$. Here we have used the chevronbracket notation introduced in Eq. (1) to denote the spatial average. The quantities $\mathcal{N}_{v}$ are real normalization constants, which depend on the choice of eigenfunctions $\boldsymbol{m}_{v}$. If the equilibrium magnetization $\hat{\boldsymbol{u}}$ corresponds to a (local) minimum of the energy, then the operator $\widehat{\Omega}$ is positive-definite. It follows that the "physical" modes with $\omega_{\nu}>0$ have positive norm
$\mathcal{N}_{v}>0$. In this formalism, the eigenfrequencies $\omega_{\nu}$ can be calculated as

$$
\omega_{\nu}=\frac{\left\langle\overline{\boldsymbol{m}}_{v} \cdot \widehat{\boldsymbol{\Omega}} * \boldsymbol{m}_{v}\right\rangle}{\mathcal{N}_{v}} .
$$

The importance of this relation is that the frequencies $\omega_{\nu}$ calculated using Eq. (8) are variationally stable with respect to perturbations of the mode profile $\boldsymbol{m}_{v}$. Thus, injecting some trial vectors inside Eq. (8) allows one to get approximate values of $\omega_{v}$ with high accuracy. ${ }^{64}$ The trial vectors should obey some simple properties: (i) They should form a complete basis in the space of vector functions $\boldsymbol{m}$; (ii) they should be locally orthogonal to $\hat{\boldsymbol{u}}$; and (iii) they should satisfy appropriate boundary conditions at the edges of the magnetic body. ${ }^{65}$

\section{Normally magnetized disks}

In this part, we establish a SW modes basis $\boldsymbol{m}_{v}$ for a normally magnetized disk. A specific feature of the considered geometry is its azimuthal symmetry. Mathematically, this means that the operator $\hat{\boldsymbol{u}} \times \widehat{\boldsymbol{\Omega}}$ commutes with the operator $\widehat{\boldsymbol{R}}_{z}$ that describes an infinitesimal rotation about the $\hat{z}$ axis, assuming that the boundary conditions are invariant under such a rotation.

This particular configuration allows us to classify the SW modes according to their behavior under the rotations in the $(x, y)$ plane. Namely, SW eigenmodes are also eigenfunctions of the operator $\widehat{\boldsymbol{R}}_{z}$ corresponding to a certain integer azimuthal number $\ell$ :

$$
\frac{\partial \boldsymbol{m}}{\partial \phi}-\hat{z} \times \boldsymbol{m}=-i(\ell-1) \boldsymbol{m} .
$$

Here $\phi$ is the azimuthal angle of the polar coordinate system.

As one can see, Eq. (9) determines the vector structure of SW modes and their dependence on the angle $\phi$. Namely, Eq. (9) for a fixed $\ell$ has two classes of solutions:

and

$$
\boldsymbol{m}_{\ell}^{(1)}=\frac{1}{2}(\hat{\boldsymbol{x}}+i \hat{\boldsymbol{y}}) e^{-i \ell \phi} \psi_{\ell}^{(1)}(\rho),
$$

$$
\boldsymbol{m}_{\ell}^{(2)}=\frac{1}{2}(\hat{\boldsymbol{x}}-i \hat{\boldsymbol{y}}) e^{-i(\ell-2) \phi} \psi_{\ell}^{(2)}(\rho),
$$

where the functions $\psi_{\ell}^{(1,2)}(\rho)$ describe the dependence of the SW mode on the radial coordinate $\rho$ and have to be determined from the dynamical equations of motion. So, the azimuthal symmetry allows one to reduce the $2 \mathrm{D}(\rho$ and $\phi)$ vector equations to a $1 \mathrm{D}(\rho)$ scalar problem.

Generally speaking, SW eigenmodes are certain linear combinations of both possible $\ell$ forms [Eqs. (10)]. The coupling of these two forms is due solely to the inhomogeneous dipolar interaction. In our experimental case (lowest-energy modes of a relatively thin disk) one can completely neglect this coupling ${ }^{66}$ and consider only the right-polarized form Eq. (10a). In the following we drop the superscript (1) in $\boldsymbol{m}_{\ell}^{(1)}$ and $\psi_{\ell}^{(1)}$.

We now find an appropriate set of radial functions $\psi_{\ell}(\rho)$ to calculate the SW spectrum using Eq. (8). Here we can take advantage of the variational stability of Eq. (8) and, instead of the exact radial profiles $\psi_{\ell}(\rho)$ (to find them one has to solve integro-differential equations), use some reasonable set of functions. Namely, it is known that the dipolar interaction in thin disks or prisms does not change qualitatively the profile 

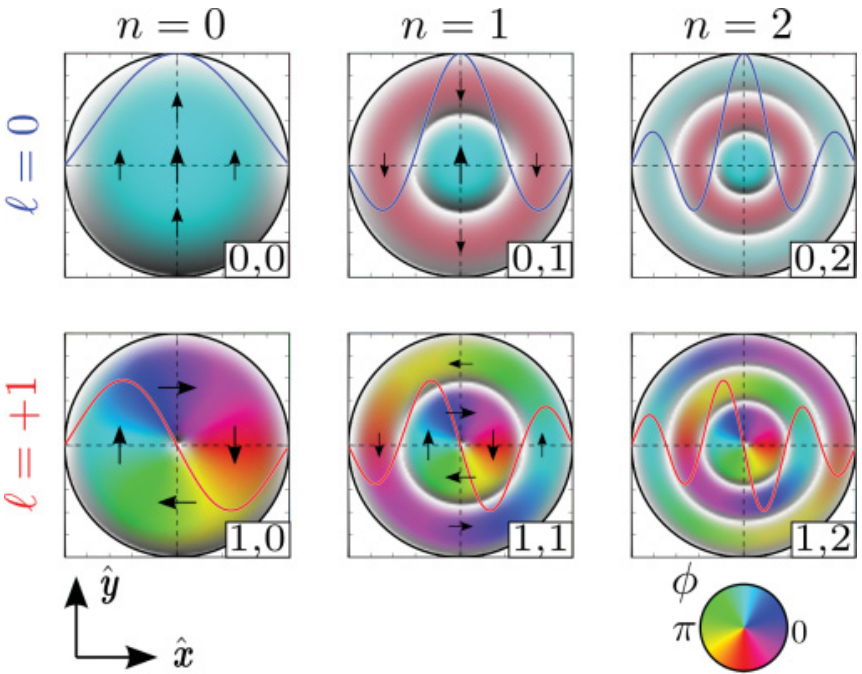

FIG. 6. (Color online) Color representation of the Bessel spatial patterns for different values of the azimuthal mode index $\ell$ (by row) and radial mode index $n$ (by column). The arrows are a snapshot of the transverse magnetization $\boldsymbol{m}_{v}$, labeled by the index $v=\ell, n$. All arrows are rotating synchronously in plane at the SW eigenfrequency. In our coding scheme, the hue indicates the phase $\phi=\arg \left(\boldsymbol{m}_{v}\right)$ (or direction) of $\boldsymbol{m}_{v}$, and the brightness the amplitude of $\left|\boldsymbol{m}_{v}\right|^{2}$. The nodal positions $\left(\left|\boldsymbol{m}_{v}\right|=0\right)$ are marked in white.

of SW modes, but introduces effective pinning at the lateral boundaries. ${ }^{65}$ Therefore, we use radial profiles of the form $\psi_{\ell}(\rho)=J_{\ell}\left(k_{\ell, n} \rho\right)$, where $J_{\ell}(x)$ is the Bessel function and $k_{\ell, n}$ are SW wave numbers determined from the pinning conditions at the disk boundary $\rho=R$. For our experimental conditions $\left(t_{a}, t_{b} \ll R\right)$, the pinning is almost complete, and we shall use $k_{\ell, n}=\kappa_{\ell, n} / R$, where $\kappa_{\ell, n}$ is the $n$th root of the Bessel function of the $\ell$ th order.

Figure 6 shows a color representation of the Bessel spatial patterns for different values of the index $v=\ell, n$. We restrict the number of panels to two values of the azimuthal mode index, $\ell=0,+1$, with the radial index varying between $n=0,1,2$. In our color code, the hue indicates the phase (or direction) of the transverse component $\boldsymbol{m}_{v}$, while the brightness indicates the amplitude of $\left|\boldsymbol{m}_{v}\right|^{2}$. The nodal positions are marked in white. A node is a location where the transverse component vanishes; that is, the magnetization vector is aligned along the equilibrium axis. This coding scheme provides a distinct visualization of the phase and amplitude of the precession profiles. The black arrows are a snapshot of the $\boldsymbol{m}_{v}$ vectors in the disk and are all rotating synchronously in plane at the SW eigenfrequency.

The top left panel shows the $v=0,0(\ell=0, n=0)$ mode, also called the uniform mode. It usually corresponds to the lowest energy mode since all the vectors are pointing in the same direction at all times. Below is the $\ell=+1, n=0$ mode. It corresponds to SWs that are rotating around the disk in the same direction as the Larmor precession. The corresponding phase is in quadrature between two orthogonal positions and this mode has a node at the center of the disk. The variation upon the $n=0,1,2$ index ( $\ell$ being fixed) shows higher-order modes with an increasing number of nodal rings. Each ring separates regions of opposite phase along the radial direction.
All these spatial patterns preserve the rotation invariance symmetry.

\section{Selection rules}

Using the complete set of Bessel functions in Eq. (8), one can obtain analytically the discrete spectrum of eigenvalues for both the thin and the thick layers. The details of the numerical application can be found in Appendix A 2. The spectral values are displayed in Fig. 7 using vertical ticks labeled $v=j_{\ell n}$, where $j=a, b$ indicates the precessing layer, and $\ell, n$ the azimuthal and radial mode indices. They are calculated at fixed applied field $H_{\text {fix }}=10 \mathrm{kOe}$ and placed on the graphs according to the frequency scale below Fig. 7(b), which is in correspondence with the field scale above Fig. 7(a) (see Sec. III C for the equivalence between field- and frequencysweep experiments).

The comparison with the experimental data in Figs. 2(a) and 2(b) shows that the coupling to an external coherent source depends primarily on the $\ell$ index. Indeed, this index carries the discriminating symmetry in SW spectroscopy. ${ }^{67}$ This is because the excitation efficiency is proportional to the overlap integral

$$
h_{v}=\frac{\left\langle\overline{\boldsymbol{m}}_{v} \cdot \boldsymbol{h}_{1}\right\rangle}{\mathcal{N}_{v}}
$$

where $\boldsymbol{h}_{1}(\boldsymbol{r})$ is the spatial profile of the external excitation field. It can be easily shown that a uniform rf magnetic field, $\boldsymbol{h}_{1}=h_{\mathrm{rf}} \boldsymbol{x}$, can only excite $\ell=0 \mathrm{SW}$ modes. We have shown in
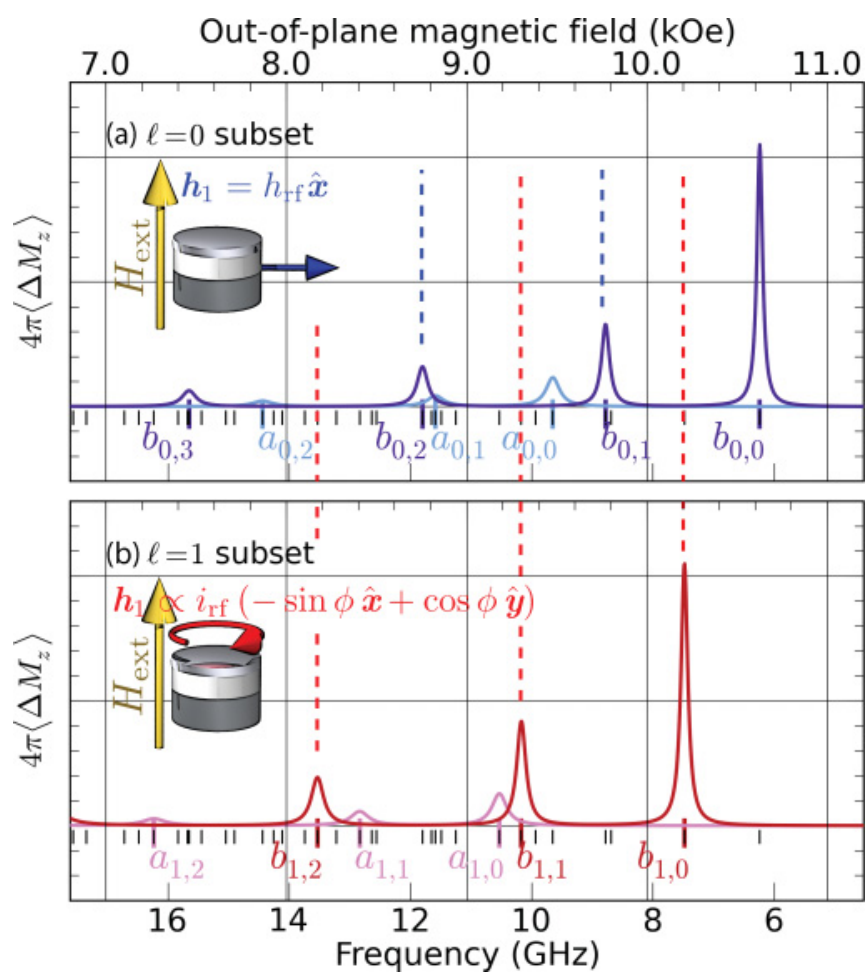

FIG. 7. (Color online) Analytically calculated spectra at $H_{\text {fix }}=$ $10 \mathrm{kOe}$ using the set of Bessel functions (see Fig. 6) as the trial eigenvectors. Panel (a) shows the linear response to a uniform excitation field $\hat{\boldsymbol{h}}_{1}=\hat{\boldsymbol{x}}$ and panel (b) to an orthoradial excitation field $\hat{\boldsymbol{h}}_{1}=-\sin \phi \hat{\boldsymbol{x}}+\cos \phi \hat{\boldsymbol{y}}$. A light (dark) color is used to indicate the energy stored Eq. (12) in the thin $\mathrm{Py}_{a}$ and thick $\mathrm{Py}_{b}$ layers. 
Fig. 7(a) the predicted position of these modes with blue-tone ticks. Obviously, the largest overlap is obtained with the so-called uniform mode $(n=0)$. Higher radial index modes $(n \neq 0)$ still couple to the uniform excitation but with a strength that decreases as $n$ increases. ${ }^{37,68}$ The $\ell \neq 0$ normal modes, however, are hidden because they have strictly no overlap with the excitation. The comparison with the experimental spectrum in Fig. 2(a) confirms that conventional FMR ${ }^{69}$ probes only partially the possible SW eigenmodes, along the $\ell=0$ index value. In contrast, the rf current-created Oersted field, $\boldsymbol{h}_{1}=$ $h_{\mathrm{Oe}}(\rho)(-\sin \phi \hat{\boldsymbol{x}}+\cos \phi \hat{\boldsymbol{y}})$ has an orthoradial symmetry and can only excite $\ell=+1 \mathrm{SW}$ modes. We have shown in Fig. 7(b) the predicted position of these modes with red-tone ticks. They are in good agreement with the resonance positions observed in Fig. 2(b). We also note that the $\ell=0$ and $\ell=+1$ spectra calculated analytically bear similar $a / b$ and $n$ index series as a function of energy. This explains why the two spectra in Figs. 2(a) and 2(b) look in translational correspondence with each other. We emphasize that the same translational correspondence would have been observed for any higher azimuthal order $\ell>1$ index spectra.

From the coupling to the excitation field expressed by Eq. (11), one can also calculate the mechanical-FMR signal $\propto\left\langle\Delta M_{z}\right\rangle$, proportional to the energy stored in the magnetic system. ${ }^{39,46}$ For an arbitrary pulsation frequency $\omega$,

$$
4 \pi\langle\Delta \boldsymbol{m} \cdot \hat{\boldsymbol{u}}\rangle \simeq 4 \pi M_{s} \sum_{v} \frac{\gamma^{2}\left|h_{v}\right|^{2}}{\left(\omega-\omega_{v}\right)^{2}+\Gamma_{v}^{2}} \mathcal{N}_{v},
$$

where the SW damping rate $\Gamma_{v}$ is given by Eq. (A8) in Appendix A 1. Equation (12) is derived under the approximation that the only relevant coefficients in the damping matrix are the diagonal terms. It has been used to compute the relative peak amplitudes in the analytically calculated spectra of Fig. 7.

\section{Comparison with experiments}

The analytical model outlined in Secs. IV A 1 and IV A 2 can be used to analyze the experimental spectra of Fig. 2, and to extract some useful parameters of the nanopillar. More details can be found in Appendix A 2 along with an approximate expression for the SW frequencies in the form of Kittel's traditional formula (with renormalized values of the effective self-demagnetization fields). This Kittel's formula, derived for the $\ell=0$ spectrum, should be used to analyze the SW spectrum excited by a uniform rf field to yield the correct values of the magnetization in our nanopillar. Identifying the experimental peaks at $H_{3}$ and $H_{(1)}$ as the lowest energy modes of the thin $\mathrm{Py}_{a}$ and thick $\mathrm{Py}_{b}$ layers yields their respective magnetizations $4 \pi M_{a}=8.0 \times 10^{3} \mathrm{G}$ and $4 \pi M_{b}=9.6 \times 10^{3} \mathrm{G}$ [see Eq. (A32)]. These values have been reported in Table I, together with those measured in the reference film (see Appendix B 3). The magnetizations extracted in the nanopillar are the same as in the extended film. The only small difference concerns the magnetization of the thin layer, which is $200 \mathrm{G}$ lower in the nanostructure than in the reference film (where $4 \pi M_{a}=8.2 \times 10^{3} \mathrm{G}$ ). We attribute this to some interdiffusion between $\mathrm{Py}$ and $\mathrm{Cu}$ or $\mathrm{Au}$ at the interfaces of the thin layer, which can happen during the etching process of the nanopillar.
Second, the separation between SW modes crucially depends on the lateral confinement in the nanopillar and thus on the precise value of its radius. Experimentally, the measured field separation between the two first peaks in Fig. 2(a) [Fig. 2(b)], which differ by an additional node in the radial direction, is $H_{(1)}-H_{(2)}=1.04 \mathrm{kOe}\left(H_{\mathbf{c}}-H_{\boldsymbol{Q}}=1.05 \mathrm{kOe}\right)$. Using the nominal radius $100 \mathrm{~nm}$ in the analytical model predicts that consecutive $n$-index modes $(n=0$ and $n=1$ modes) should be separated by $1.33 \mathrm{kOe}$, which is larger than the observed value. This separation drops to $1.05 \mathrm{kOe}$ for a larger disk radius $R=125 \mathrm{~nm}$, which we thus refer to as the radius of our nanopillar. This value of $R$ also makes it possible to estimate the shift between the $\ell=0$ and $\ell=+1$ spectra, found to be $530 \mathrm{Oe}$, in good agreement with the experimental value $H_{(1}-H_{\mathbf{0}}=470$ Oe observed in Fig. 2.

\section{B. Influence of dipolar coupling between different layers}

In the treatment above we have neglected the dynamic coupling between the two magnetic disks in dipolar interaction. In general, the interaction between two identical magnetic layers will lead to the hybridization of the same $v$-index mode of each layer into two collective modes: the acoustic mode, where the layers are precessing in phase, and the optical mode, where they are precessing in antiphase. This has been observed in interlayer-exchange-coupled thin films ${ }^{70}$ and in trilayered wires where the two magnetic stripes are dipolarly coupled. ${ }^{71}$ In the case where the two magnetic layers are not identical (different geometry or magnetic parameters), this general picture continues to subsist. Although both isolated layers have eigenmodes with different eigenfrequencies, the collective magnetization dynamics still splits into a binding state and an antibinding state. However, here the precession of magnetization can be more intense in one of the two layers and the spectral shift of the coupled SW modes with respect to the isolated SW modes is reduced, as it was observed in both the dipolarly ${ }^{71}$ and exchange-coupled cases. ${ }^{72}$

Here we assume that the dominant coupling mechanism between the Py layers is the magnetic dipolar interaction. We neglect any exchange coupling between the magnetic layers mediated through the normal spacer or any coupling associated with pure spin currents ${ }^{14}$ in our all-metallic spinvalve structure. To analyze the influence of the dipolar coupling between the two magnetic layers, one can complement the perturbation theory derived in Sec. IV A and in Appendix A 1. Denoting $c_{j}$ the SW amplitudes in $j$ th disk, one can get from Eq. (A6):

$$
\begin{aligned}
& \frac{d c_{a}}{d t}=-i \omega_{a} c_{a}+i \gamma h_{a, b} c_{b}, \\
& \frac{d c_{b}}{d t}=-i \omega_{b} c_{b}+i \gamma h_{b, a} c_{a},
\end{aligned}
$$

where $\omega_{j}$ is the frequency of the $j$ th disk $(j=a, b)$ with account of only the static field of the $j^{\prime}$ th $\operatorname{disk}\left(j^{\prime}=b, a\right)$ (i.e., with $\boldsymbol{m}_{j}^{\prime}$ fixed at equilibrium; see Fig. 8). The cross term $h_{j, j^{\prime}}$ is given by

$$
h_{j, j^{\prime}}=-\frac{4 \pi M_{j^{\prime}}}{\mathcal{N}_{j}}\left\langle\overline{\boldsymbol{m}}_{j} \cdot \widehat{\boldsymbol{G}}^{(d)} * \boldsymbol{m}_{j^{\prime}}\right\rangle_{j} .
$$




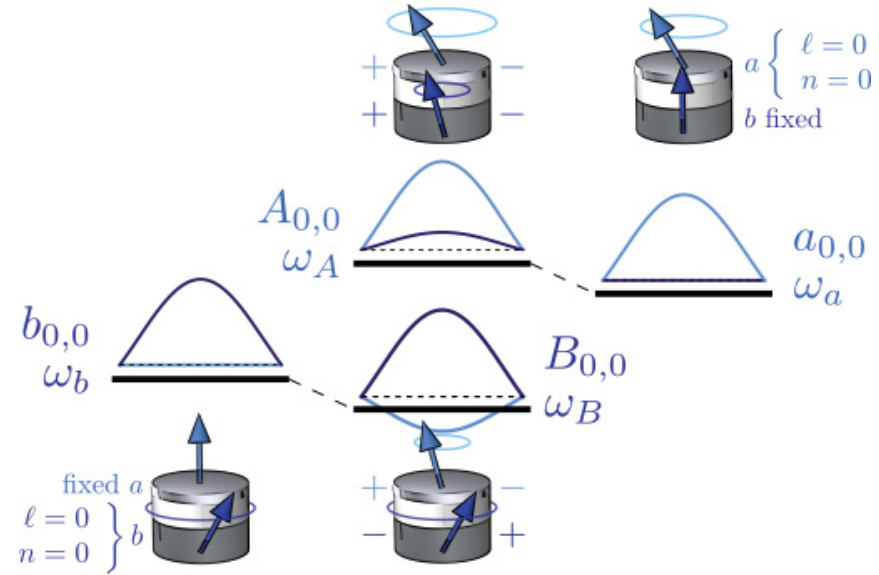

FIG. 8. (Color online) Schematic representation of the coupled dynamics between two different magnetic disks. Here, $\omega_{b}$, the eigenfrequency of the lowest energy precession mode in the thick layer (the thin layer being fixed at equilibrium) is smaller than $\omega_{a}$, the one in the thin layer (the thick layer being fixed at equilibrium). When the two disks are dynamically coupled through the dipolar interaction, the binding state $B$ corresponds to the two layers oscillating in antiphase at $\omega_{B}$, with the precession occurring mostly in the thick layer, whereas the antibinding state $A$ corresponds to the layers oscillating in phase at $\omega_{A}$, with the precession mostly in the thin layer. This is shown by displaying the dipolar charges and the precession profile $\boldsymbol{m}(\rho)$ in each layer using a light (dark) color to represent the contribution of the thin (thick) layer.

Here $\widehat{\boldsymbol{G}}^{(d)}$ represents the magnetodipolar interaction, $M_{j^{\prime}}$ is the saturation magnetization of the $j^{\prime}$ th disk, and the averaging goes over the volume of $j$ th disk. Thus, $h_{j, j^{\prime}}$ is the magnetic stray field produced by the dynamic magnetization of the $j^{\prime}$ th disk projected along the local deviation vector inside the $j$ th disk and averaged over its volume. It can be shown that the overlap defined in Eq. (14) is maximum between mode pairs bearing similar wave numbers in each layer (i.e., the same set of indices $v) .^{71}$ This is the reason why dropping the index $v$ in Eqs. (13) and (14) is a reasonable approximation.

The antibinding $(A)$ and binding $(B)$ eigenfrequencies of Eqs. (13) have the form

$$
\omega_{A, B}=\frac{\omega_{a}+\omega_{b}}{2} \pm \sqrt{\left(\frac{\omega_{a}-\omega_{b}}{2}\right)^{2}+\Omega^{2}},
$$

where

$$
\Omega^{2}=\gamma^{2} h_{a, b} h_{b, a} .
$$

In the case when the dipolar coupling is small $\left(\Omega \ll \mid \omega_{a}-\right.$ $\omega_{b} \mid$ ), the eigenfrequencies can be written as (we assume $\omega_{a}>$ $\left.\omega_{b}\right)$

$$
\begin{aligned}
& \omega_{A}=\omega_{a}+\frac{\Omega^{2}}{\omega_{a}-\omega_{b}}, \\
& \omega_{B}=\omega_{b}-\frac{\Omega^{2}}{\omega_{a}-\omega_{b}} .
\end{aligned}
$$

These equations can be used for quantitative purposes when $\Omega /\left|\omega_{a}-\omega_{b}\right|<0.3$, in which case they describe frequency shift with accuracy better than $10 \%$. Thus, the larger of the frequencies $\left(\omega_{a}\right)$ shifts up by

$$
\Delta \omega=\frac{\Omega^{2}}{\omega_{a}-\omega_{b}},
$$

while the smaller one $\left(\omega_{b}\right)$ shifts down by the same amount. This effect is summarized in Fig. 8.

A numerical estimate of the coupling strengths $h_{a, b}$ and $h_{b, a}$ between the lowest energy SW modes in each disk can be found in Appendix A 2. The obtained result is very close to the approximate estimation used in Ref. 73, where the spatial structure of the interacting SW modes is ignored to calculate the dipolar coupling between uniformly precessing disks. For the experimental parameters, $\Omega / 2 \pi \simeq 0.5 \mathrm{GHz}$. This coupling is almost an order of magnitude smaller than the frequency splitting $\omega_{a}-\omega_{b}$, caused, mainly, by the difference of effective magnetizations of two disks: $\gamma 4 \pi\left(M_{b}-M_{a}\right) \simeq 2 \pi \cdot 4.5 \mathrm{GHz}$. As a result, the shift of the resonance frequencies due to the dipolar coupling is negligible, $\Delta \omega / 2 \pi \simeq 0.06 \mathrm{GHz}$.

Using Eqs. (13), one can also estimate the level of mode hybridization due to the dipolar coupling. For instance, at the frequency $\omega_{A} \approx \omega_{a}$, the ratio between the precession amplitudes in the two layers is given by

$$
\left|c_{b} / c_{a}\right|_{\omega_{A}}=\Delta \omega /\left(\gamma h_{a, b}\right) \simeq \frac{\Omega}{\omega_{a}-\omega_{b}} .
$$

For the experimental parameters, $\Omega /\left(\omega_{a}-\omega_{b}\right) \approx 0.1$; that is, the precession amplitude in the disk $b$ is about $10 \%$ of that in the disk $a$. Thus, although the dipolar coupling induces a small spectral shift [second order in the coupling parameter, Eq. (19)], its influence in the relative precession amplitude is significant [first order in the coupling parameter; Eq. (20)]. Finally, we point out that here the dipolar coupling is antiferromagnetic and that the binding (lower energy) mode $B$ always corresponds to the thick layer mainly precessing, with the thin layer vibrating in antiphase, and vice versa for the antibinding (in-phase) mode $A$ (see dipolar charges in Fig. 8).

\section{Micromagnetic simulations}

In the analytical formalism presented above, several approximations have been made. For instance, we have assumed total pinning at the disks boundary for the SW modes and no variation of the precession profile along the disks thicknesses (2D model), and we have neglected the dependence on $v$ of the dynamic dipolar coupling. Still, it makes it possible to extract important parameters in our nanopillar, such as its radius and the magnetization in both layers. It also describes the influence of the dynamic dipolar coupling on the position and collective character of the SW modes.

Instead of developing a more complex analytical formalism, we have performed innovative 3D micromagnetic simulations in order to go beyond the approximations mentioned above and to unambiguously identify the SW modes observed in our nanopillar sample. For that purpose, we have used a combination of micromagnetic simulation solvers available as part of SpinFlow 3D, a finite element based simulation platform for spintronics developed by In Silicio. ${ }^{74}$ The steady-state micromagnetic solver used to obtain numerical approximations of micromagnetic equilibrium states is based on a weak 
formulation and Galerkin-type finite element implementation of the very efficient projection scheme introduced in Ref. 75 . A second numerical solver, a micromagnetic eigensolver, has been used for fast calculations of lossless 3D SW eigenmodes. It is based on a finite element discretization of the generalized eigenvalue problem defined by the linearized lossless magnetization dynamics in the vicinity of an arbitrary precomputed equilibrium state, following an approach very similar to the one introduced in Ref. 76. The discrete generalized eigen-value problem is solved with an iterative Arnoldi method using the ARPACK library. ${ }^{77}$ In this calculation the full complexity of the 3D micromagnetic dynamics of the presently considered bilayer system is preserved. The solver outputs both the eigenvalues by increasing energy order and the associated eigenvectors. Several tens of SW eigenmodes can be accurately computed in a matter of few minutes of CPU time with a standard desktop PC, for magnetic thin-film nanostructures with typical lateral sizes in the $100-\mathrm{nm}$ range. This is two to three orders of magnitude faster compared to the required computation time when using more traditional approaches for micromagnetic computation of SW eigenmodes, which are typically based on the Fourier component analysis of time series generated by the solution of the full nonlinear Landau-Lifshitz-Gilbert equation. ${ }^{78}$ Finally, a quite generic linear response solver, implementing among other things the spectral decomposition of the MRFM signal as expressed in Eqs. (11), (12), and (A8), has been used to compute the MRFM spectra shown here.

To proceed, the nanopillar is first discretized using unstructured meshing algorithms resulting in an average mesh size of $3.5 \mathrm{~nm}$. This corresponds to a total number of vertices in the vicinity of $5 \times 10^{4}$. The magnetization vector is interpolated linearly inside each cell (tetrahedra), a valid approximation taking into account that the cell sizes are smaller than the exchange length $\Lambda_{\mathrm{ex}} \simeq 5 \mathrm{~nm}$ in permalloy. The magnetic parameters introduced in the code are the ones reported in Table I, and the simulation incorporates the perturbing presence of the magnetic sphere attached on the cantilever. Moreover, the 10-nm-thick $\mathrm{Cu}$ spacer is replaced by vacuum, so that the layers are only coupled through the dipolar interaction (spin diffusion effects are absent).

The next step is to calculate the equilibrium configuration in the nanopillar at $H_{\mathrm{ext}}=H_{\mathrm{fix}}=10 \mathrm{kOe}$. The external magnetic field is applied exactly along $\hat{z}$ and the spherical probe with a magnetic moment $m=2 \times 10^{-10} \mathrm{emu}$ is placed on the axial symmetry axis at a distance $s=1.3 \mu \mathrm{m}$ above the upper surface of the nanopillar. The convergence criterion introduced in the code is $\left|d M_{z} / M_{j}\right|<2 \times 10^{-9}$ between iterations. The result shown in the inset of Fig. 5 reveals that the equilibrium configuration is almost uniformly saturated along $\hat{z}$. Still, a small tilt $\left(<5^{\circ}\right)$ of the magnetization, away from $\hat{z}$ and along the radial direction, is observed at the periphery of the thick and thin layers.

The micromagnetic eigensolver is then used to compute the lowest eigenvalues of the problem as well as the associated eigenvectors. The discrete list of eigenvalues under $18 \mathrm{GHz}$ is shown as black vertical ticks at the bottom of Figs. 9(a) and 10(a). The precession patterns of the six eigenvectors corresponding to the six lowest eigenfrequencies are shown in Fig. 11. The middle and right columns show the dynamics
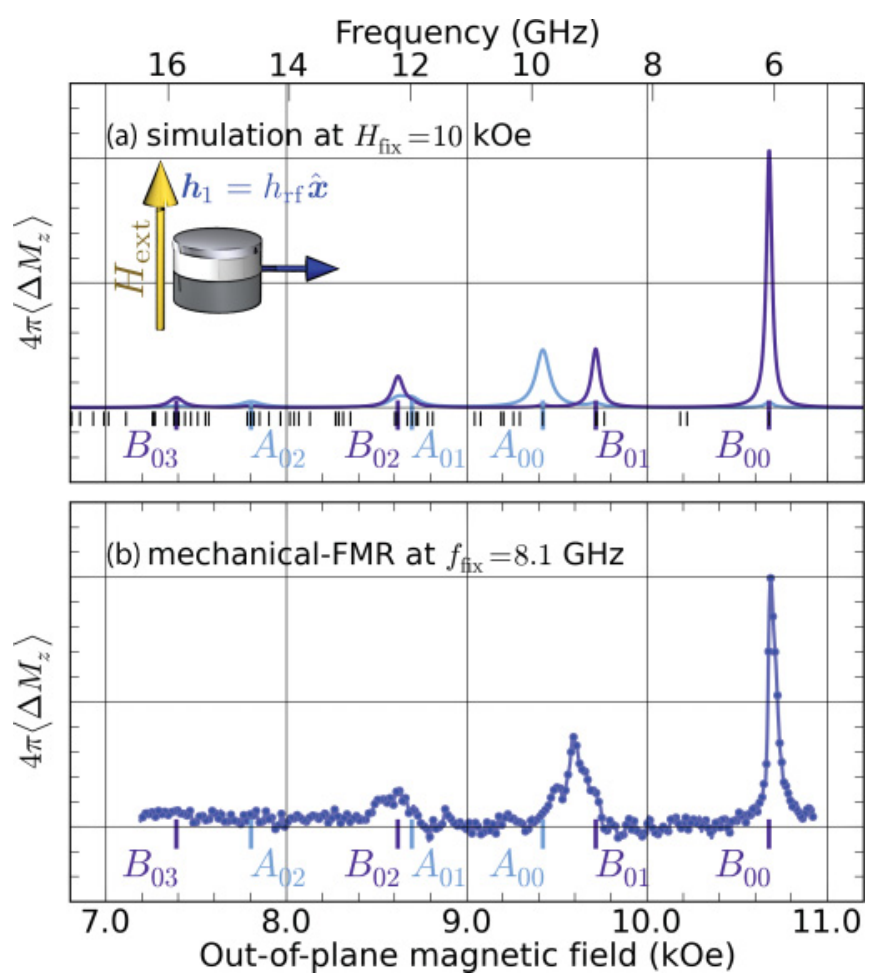

FIG. 9. (Color online) Panel (a) is the numerically calculated spectral response to a uniform excitation field $\boldsymbol{h}_{1} \propto \hat{\boldsymbol{x}}$, from a 3D micromagnetic simulation performed at $H_{\text {fix }}=10 \mathrm{kOe}$. The peaks are labeled according to their precession profiles shown in Fig. 11. A light (dark) color is used to indicate the energy stored in the thin (thick) layer. Panel (b) recalls the experimental spectrum measured by mechanical FMR when exciting the nanopillar by a homogeneous rf magnetic field at $f_{\text {fix }}=8.1 \mathrm{GHz}$.

$\boldsymbol{m}$ in the thin $\mathrm{Py}_{a}$ and thick $\mathrm{Py}_{b}$ layers, while the precession profiles along the median direction are shown on the left in light and dark colors, respectively. The resonance peaks are labeled according to the SW modes precession profiles and the eigenvalues of the simulated peaks are reported in Table II.

From the eigenvectors' spatial patterns, one can compute their coupling [Eq. (11)] to a uniform rf field $\boldsymbol{h}_{1}=h_{\mathrm{rf}} \hat{\boldsymbol{x}}$ and, with Eq. (12), the mechanical-FMR spectrum [Fig. 9(a)]. The same procedure is repeated for the rf current-induced Oersted field $\boldsymbol{h}_{1} \propto i_{\mathrm{rf}}(-\sin \phi \hat{\boldsymbol{x}}+\cos \phi \hat{\boldsymbol{y}})$ excitation [Fig. 10(a)]. Since the code gives access to the contribution of each layer, a light (dark) tone is used to indicate the vibration amplitude in the thin (thick) layer in the two figures. For comparison, the mechanical-FMR spectra of Figs. 2(a) and 4(a) have been reported in Figs. 9(b) and 10(b), respectively. We have applied the same conversion between the frequency (top) and field (bottom) scales as discussed in Sec. III C.

In Fig. 9(a), the largest peak in the simulation occurs at the same field as the experimental peak at $H_{\triangleright}$. This lowest energy mode corresponds to the most uniform mode with the largest wave vector and no node along the radial direction; thus, it has the index $n=0$. It has uniform phase along the azimuthal direction, which is the character of the $\ell=0$ index. For this mode, the thick layer is mainly precessing, with the thin layer oscillating in antiphase (binding index $B$ ), as can be 

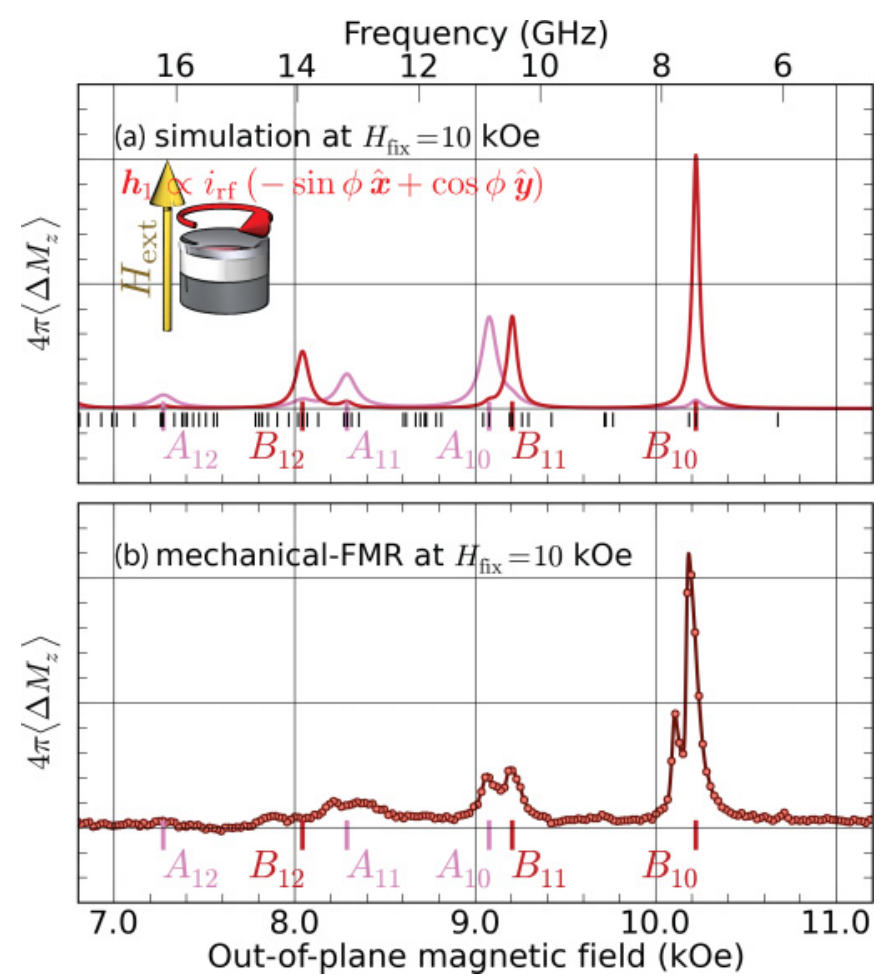

FIG. 10. (Color online) Panel (a) is the simulated spectral response to an orthoradial excitation field $\boldsymbol{h}_{1} \propto-\sin \phi \hat{\boldsymbol{x}}+\cos \phi \hat{\boldsymbol{y}}$. Panel (b) recalls the experimental spectrum measured by mechanicalFMR for an rf current excitation.

seen from its spatial profile in Fig. 11. The same analysis can be made for the second peak, labeled $B_{01}$, which occurs close to the peak at $H_{\odot}$. It also corresponds to a resonance mainly of the thick layer, and its color representation shows that this is the first radial harmonic $(n=1)$, with one line of nodes in the radial direction. Again, the thin layer is oscillating in antiphase, with the same radial index $n=1$, as clearly shown by the mode profile along the median direction. The third peak is labeled $A_{00}$ and is located close to the experimental peak at $H_{3}$. It corresponds this time to a uniform $(n=0)$ precession mainly located in the thin layer, in agreement with the experimental analysis presented in Sec. III A. In this mode, the thick layer is also vibrating in phase with the thin layer (antibinding index $A$ ).

We can also look at the relative amplitudes of precession in the two disks to quantify the dynamic coupling between the disks. From the profiles shown in Fig. 11, one can infer that for the fundamental mode $B_{00}$, the amplitude of precession is distributed with a ratio of about 3:1 between the thick (75\%) and the thin layers $(25 \%)$. For the mode $A_{00}$, the ratio is $8: 1$ in favor of the thin layer, which contributes to $89 \%$ of the precession amplitude (11\% for the thick layer). These relative precession amplitudes were expected from the relative weight of the thick and thin layers and from the approximate analytical model presented in Sec. IV B. The simulated field separation between the two coupled uniform modes $\left(\omega_{B_{00}}-\omega_{A_{00}}\right) / \gamma=1.28 \mathrm{kOe}$ compares also well with the $1.30 \mathrm{kOe}$ estimate from the $2 \mathrm{D}$ model, with the dynamic dipolar coupling taken into account. Finally, one can check from the simulations the independence of the precession

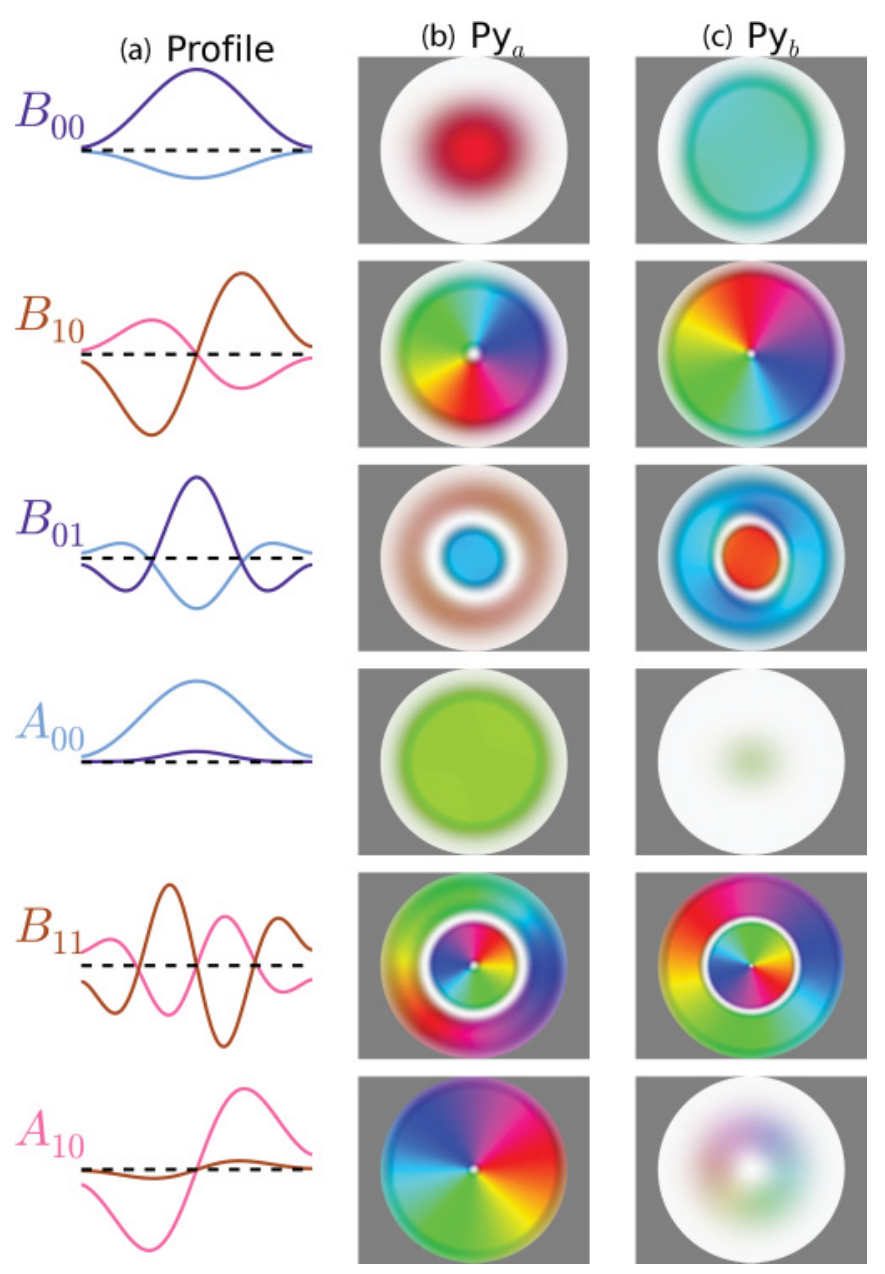

FIG. 11. (Color online) Simulated precession patterns of the eigenvectors. Column (a) shows the precession profiles across the thin (light color) and thick (dark color) layers. Columns (b) and (c) show the dynamics in the thin $\mathrm{Py}_{a}$ and thick $\mathrm{Py}_{b}$ layers, respectively, with the color code defined in Fig. 6.

profiles on the thickness. This confirms the validity of the 2D approximation and explains the performances of the analytical model.

TABLE II. Comparative table of the resonance values for the SW modes, arranged in order of increasing energy. On the left are the consecutive peak locations measured experimentally. Experiments are performed at $f_{\text {fix }}=8.1 \mathrm{GHz}$ [Fig. 2] or $H_{\text {fix }}=10 \mathrm{kOe}$ [Fig. 4(a)]. On the right are the simulated eigenfrequencies $f$ at $H_{\text {fix }}=10 \mathrm{kOe}$. The conversion to field value $H_{\text {ext }}$ is obtained through $H_{\text {ext }}-H_{\text {fix }}=$ $2 \pi\left(f-f_{\text {fix }}\right) / \gamma$.

\begin{tabular}{lcrccc}
\hline \hline Exp. & $f(\mathrm{GHz})$ & $H_{\text {ext }}(\mathrm{kOe})$ & Simulated & $f(\mathrm{GHz})$ & $H_{\mathrm{ext}}(\mathrm{kOe})$ \\
\hline (1) & & 10.69 & $B_{00}$ & 6.08 & 10.68 \\
0 & \multirow{2}{*}{7.37} & 10.22 & $B_{10}$ & 7.44 & 10.22 \\
(2) & & 9.65 & $B_{01}$ & 8.95 & 9.71 \\
(3) & & 9.51 & $A_{00}$ & 9.82 & 9.42 \\
(2) & 10.48 & 9.17 & $B_{11}$ & 10.47 & 9.20 \\
3 & 10.92 & 9.07 & $A_{10}$ & 10.85 & 9.08 \\
(4) & \multirow{2}{*}{13.41} & 8.64 & $A_{01}$ & 11.98 & 8.69 \\
(4 & 8.22 & $A_{11}$ & 13.19 & 8.29 \\
\hline \hline
\end{tabular}


We now briefly comment on the simulated spectrum of Fig. 10, which makes it possible to identify the SW modes excited by the orthoradial Oersted field produced by the rf current flowing through the nanopillar. From Figs. 10 and 11, it is clear that the modes which couple to this excitation symmetry have a rotating phase in the azimuthal direction, characteristic of the $\ell=+1$ modes. ${ }^{79}$ We find that the SW modes of Fig. 10 show the same series of $A / B$ and $n$ indices as those in Fig. 9 (but their $\ell$ index is different). This sustains the translational correspondence between the SW spectra of Figs. 2(a) and 2(b). Finally, we point out that, for all the modes displayed in Fig. 11, the pinning conditions at the boundaries of each disk are not trivial, which we attribute to the collective nature of the motion driven by the dipolar coupling. ${ }^{80}$ The general trend observed here is that the thin layer is less pinned than the thick layer for in-phase modes, and vice versa.

To summarize, the 3D micromagnetic simulations enable the identification (with three indices, $A / B, \ell$, and $n$ ) of the SW modes probed experimentally by both a uniform rf magnetic field and a rf current flowing through the nanopillar, that is, of their respective selection rules. They confirm the experimental analysis performed in Sec. III and give a deeper insight on the collective nature of the magnetization dynamics in the nanopillar discussed in Sec. IV B.

\section{SYMMETRY BREAKING}

In the following, we review some characteristic spectral features associated with the breaking of the axial symmetry in our experiment. First, we experimentally report on the appearance of $\ell=0$ modes in the SW spectrum excited by an rf current flowing through the nanopillar, when a small tilt angle is introduced between the applied field and the normal of the layers. This bridges the gap between our mechanical-FMR experiments and usual ST-FMR measurements. ${ }^{25,26}$ Second, we have simulated the spectral distortions introduced by breaking the cylindrical symmetry of the SW confinement potential. This makes it possible to explain the lift of degeneracy in the SW spectrum, which leads to the splitting of modes.

\section{A. Polar angle dependence}

The dependence on the polar angle $\theta_{H}=\left(\hat{z}, \boldsymbol{H}_{\mathrm{ext}}\right)$ of the mechanical-FMR spectra excited by a uniform rf magnetic field and by an rf current flowing through the nanopillar is presented in Fig. 12. Let us first focus on the conventional FMR spectra shown in Fig. 12(a), acquired at three different polar angles from the exact perpendicularity, increasing by steps of $1^{\circ}$. The main effect here is the shift of the $\ell=0 \mathrm{SW}$ modes spectrum toward lower field as $\theta_{H}$ increases, which has been explained in details in Ref. 38 for a single magnetic disk. It is due to the decrease of the demagnetizing field produced by the tilt of the equilibrium magnetization away from the normal. In fact, in each magnetic layer $j=a, b$, the uniform magnetization creates a nonuniform dipolar field $4 \pi M_{j} \widehat{\boldsymbol{G}}^{\left(d_{j}\right)} *$ $\hat{\boldsymbol{u}}_{j}$, which is maximum in the exact normal configuration. The equilibrium direction $\hat{\boldsymbol{u}}_{j}$ is in the plane $\left(\hat{\boldsymbol{z}}, \boldsymbol{H}_{\text {ext }}\right)$ and makes a polar angle $\theta_{j}>\theta_{H}$ with the normal determined by Eq. (A34). It can be estimated that when $H_{\mathrm{ext}} \approx 10 \mathrm{kOe}$ and $\theta_{H}$ increases from $0^{\circ}$ to $2^{\circ}$, the equilibrium angles $\theta_{a}$ and $\theta_{b}$ of the static

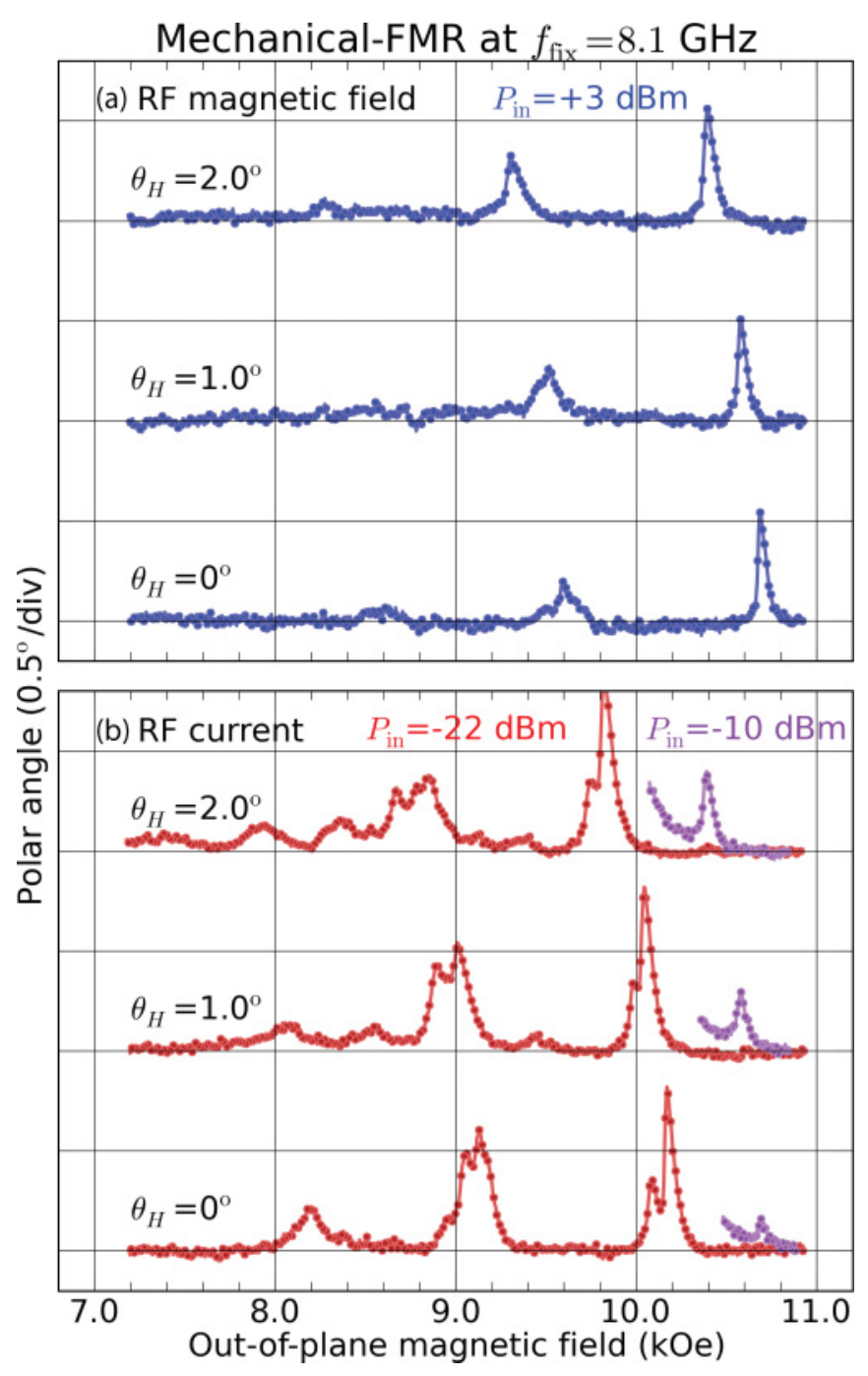

FIG. 12. (Color online) Dependence of the mechanical-FMR spectra excited by a uniform rf magnetic field (a) and by a rf current flowing through the nanopillar (b) on the polar angle $\theta_{H}$ between the applied field and the normal to the layers. Superposed (in purple) is the behavior of the high field tail at larger power.

magnetization in the thin and thick layers linearly increases from $0^{\circ}$ to $\approx 9^{\circ}$ and from $0^{\circ}$ to $\approx 13^{\circ}$, respectively. This leads to a shift to lower field of the FMR spectrum by about $420 \mathrm{Oe}$ (see Appendix A 2), in agreement with the data. We also emphasize that, in fact, the profiles of the SW eigenmodes are affected by the breaking of axial symmetry and that the pure $\ell=0$ eigenmodes when $\theta_{H}=0$ become mixed with $\ell \neq 0$ modes $^{38}$ when $\theta_{H} \neq 0$.

We now turn to the influence of the polar angle $\theta_{H}$ on the FMR spectra excited by a rf current $\left(i_{\mathrm{rf}}=170 \mu \mathrm{A}\right)$. The same global shift toward lower field as discussed above is observed in Fig. 12(b) by looking at the red spectra acquired with an increasing $\theta_{H}$. However, there is an important additional effect here. Whereas only $\ell=+1 \mathrm{SW}$ modes are excited by the rf current flowing through the nanopillar in the exact perpendicular geometry, resonance peaks can also be detected at the positions of $\ell=0 \mathrm{SW}$ modes when $\theta_{H} \neq 0$. Although the amplitudes of the $\ell=0$ modes are not large in Fig. 12(b), it is quite clear that they all grow as $\theta_{H}$ increases. In order 
to reveal this effect better, we have reported in purple on the same figure the resonance peak of the mode $B_{00}$ excited with $\mathrm{a}+12 \mathrm{~dB}$ larger power $\left(i_{\mathrm{rf}} \simeq 680 \mu \mathrm{A}\right)$, as a function of $\theta_{H}$. Despite the large rf current excitation, its amplitude almost vanishes at $\theta_{H}=0$. Then it increases linearly with $\theta_{H}$ until it becomes almost as large as when it is excited by the uniform rf field $h_{\text {rf }} \simeq 2.1$ Oe used in Fig. 12(a).

The experimental data and their analysis presented in the previous Secs. II to IV demonstrate that in the exact perpendicular configuration, only $\ell=+1$ modes are excited by the rf current flowing through the nanopillar, due to the orthoradial symmetry of the induced rf Oersted field [Eq. (A14)]. Because there is no overlap between this particular excitation symmetry and the uniform azimuthal symmetry of the $\ell=0$ modes, the latter do not couple to the rf current excitation. The fact that these hidden modes in the exact perpendicular configuration can be excited by introducing a small misalignment angle between the applied field and the normal to the nanopillar $\hat{z}$ is a striking result. It means that the selection rules associated with the rf current excitation change if the applied field is tilted away from $\hat{z}$, which we now explain.

Due to the smaller demagnetizing field in the thin magnetic disk than in the thick one (due to $M_{a}<M_{b}$ ), the equilibrium angle of the thin layer is smaller than in the thick layer, $\theta_{a}<$ $\theta_{b}$, as obtained from Eq. (A34). For the parameters of our nanopillar, $\beta=\theta_{b}-\theta_{a} \approx 2 \theta_{H}$, at $H_{\mathrm{ext}} \approx 10 \mathrm{kOe}$ and for a small angle $\theta_{H}$. It means that if $\theta_{H} \neq 0$, the magnetization vectors in both layers are misaligned from each other by an angle $\beta=\left(\boldsymbol{M}_{a}, \boldsymbol{M}_{b}\right)$, so that the cross product $\hat{\boldsymbol{u}}_{a} \times \hat{\boldsymbol{u}}_{b}$ is finite and lies in the plane parallel to the layers, say along $\hat{\boldsymbol{x}}$. Thus, the spin transfer excitation $(2 \pi \lambda)^{-1} i_{\mathrm{rf}} \sin \beta \hat{\boldsymbol{x}}$ associated with the rf current flowing through the spin-valve nanopillar, ${ }^{25,26}$ which is vanishing in the exact perpendicular configuration where $\beta=0$, becomes finite if there is a small misalignment angle $\theta_{H} \neq 0$ [see Eqs. (A15) and (A16) in Appendix A 1, $(2 \pi \lambda)^{-1}$ is the spin transfer efficiency]. Because this so-called ST-FMR excitation has the same symmetry as an in-plane uniform rf magnetic field, it is expected to excite SW modes having the $\ell=0$ index symmetry. Still, this excitation has to compete with the rf Oersted field excitation, which is independent of $\theta_{H}$ and is much larger in our configuration due to the small value of $\beta\left(<5^{\circ}\right)$. Therefore, the amplitudes of the $\ell=+1$ modes are much larger than those of the $\ell=0$ modes in Fig. 12(b).

It is also clear that the amplitude of the mode $B_{00}$ excited by the rf current [purple peaks in Fig. 12(b)] grows linearly with $\theta_{H}$, as expected from the above discussion. We emphasize that a quantitative understanding of the amplitude of the peaks excited by ST-FMR would require to consider the collective nature of the dynamics in the nanopillar and the asymmetry of spin transfer in the thick and thin magnetic layers. Finally, we note that the small signal observed at $\theta_{H}=0$ should, in principle, vanish with the ST-FMR excitation. This reminiscent signal can be ascribed to a small misalignment of the applied field with respect to the normal to the nanopillar (the precision on the orientation is $0.2^{\circ}$ ) or to a slight asymmetry of the rf current lines through the nanopillar, which would induce a small asymmetry of the rf Oersted field, thereby adding a small in-plane uniform component to the orthoradial magnetic field.

To summarize, this study makes it possible to derive the selection rules of the rf current excitation. In the exact perpendicular configuration, the magnetizations of both layers are aligned, and only $\ell=+1$ modes can be excited due to the orthoradial symmetry of the current-created Oersted field ( $\ell=0$ modes are hidden). However, when a finite angle is introduced between the magnetizations in each layer by slightly tilting the applied field away from the normal, $\ell=0$ modes can be excited by ST-FMR, which has the same symmetry as a uniform rf field excitation polarized in plane.

\section{B. Confinement asymmetries}

As seen in Sec. IV C, the 3D micromagnetic simulations make it possible to identify the SW modes observed in the experimental spectra. Still, the latter are more rich than the simulated power spectra, due to the splitting of some resonance peaks, which was noted in Secs. II C and III A. In particular, the experimental peak at $H_{\mathbf{o}}$, identified as the mode $B_{10}$, is clearly split in two, with a smaller resonance about 100 Oe away in the low field wing of the main peak, which is not the case in the simulation (see Fig. 10). The peaks at $H_{0}$ (identified as $\left.B_{11}\right)$ and at $H_{\mathbf{3}}\left(A_{10}\right)$ are also split, contrary to the simulations, where all these peaks are single. In contrast to these observations, the peak at $H_{(1}$, which is the uniform mode $B_{00}$, is single in both the experimental and the simulated spectra.

So it seems that, experimentally, the occurrence of the mode splitting depends on the mode index, whereas in 3D simulations, in which the nanopillar has a perfect cylindrical shape, none of the resonance peaks is split. This suggests that the observed splittings are related to asymmetries in the confinement of the disks and that the various SW modes are affected differently because they probe different regions. The fact that the double peak at $H_{\mathbf{o}}$ depends on the tilt angle [see Fig. 12(b)] and is more or less pronounced depending on the direction in which the applied field is tilted from the normal (not shown) is another strong indication that some symmetry breaking in the lateral confinement is at the origin of this effect. ${ }^{81}$

To support this idea, we have carried out new 3D micromagnetic simulations with the SpinFlow 3D package on a structure that break the perfect cylindrical symmetry of the nanopillar. We have kept a perfectly flat structure, but we have used an elliptical cross-section. The long axis of the ellipse is $250 \mathrm{~nm}$, while the short axis is $200 \mathrm{~nm}$. The influence of this breaking of symmetry on, respectively, the $\ell=0$ spectrum (rf field excitation) and the $\ell=+1$ spectrum (rf current excitation) is presented in Fig. 13.

Concentrating first on the standard FMR SW spectrum of Fig. 13(a), one can see that the lowest energy mode $B_{00}$ remains a single peak. This illustrates the intuitive idea that the uniform SW mode, where the oscillation power is mostly concentrated at the center, is rather not sensitive to change of the confinement at the periphery. The same behavior applies for the lowest energy mode of the thin layer, $A_{00}$.

The simulated SW spectrum of Fig. 13(b) shows a different sensitivity to the shape asymmetry. It is observed that the lowest energy mode with the $\ell=+1$ index splits in two peaks, in contrast with the lowest energy $\ell=0$ mode, which remains a single peak. We also note that the satellite peak, induced by the elliptical confinement, is located in the low-field wing of the main resonance, as in the experiments of Fig. 2(b). 

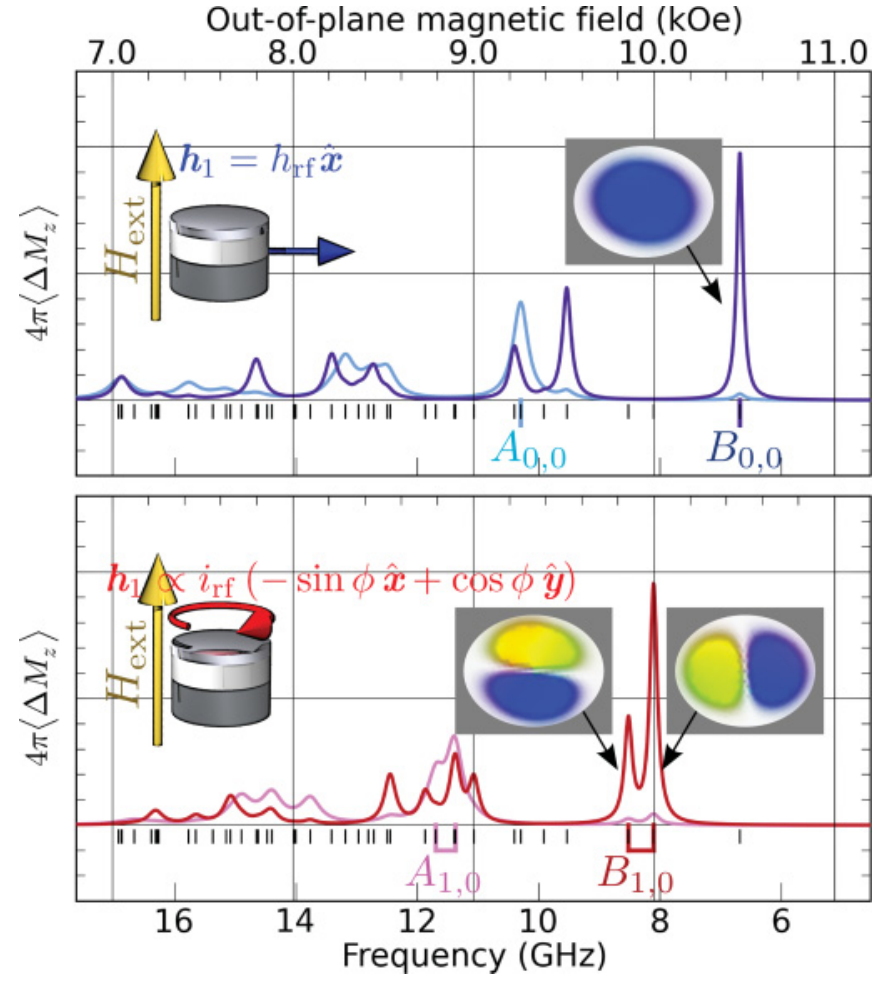

FIG. 13. (Color online) Simulated SW spectra for a nanopillar with an elliptical section (see text). Linear response to a homogeneous rf magnetic field excitation (a) and to an orthoradial rf Oersted field excitation (b). The precession patterns of the lowest energy modes are shown in the insets.

The precession patterns shown in the two insets Fig. 13(b) reveal that the elliptical shape introduces some mixing between the $\ell=+1$ and $\ell=-1 \mathrm{SW}$ modes (the $\ell=-1$ mode corresponds to SWs that are rotating around the disk in the opposite direction as the Larmor precession). In a circular disk, these two modes are degenerate, and only the $\ell=+1$ mode couples to the orthoradial Oersted field excitation. However, in the ellipse, the two eigenmodes split and become mixed, as shown by the two eigenvectors displayed in Fig. 13(b), which correspond to the linear combinations $J_{1}(\rho)+J_{-1}(\rho)$ and $J_{1}(\rho)-J_{-1}(\rho)$. The simulated spectrum of Fig. 13(b) reproduces well the main features of the mechanical-FMR spectrum of Fig. 2(b), including the splittings observed for the $\ell=+1 \mathrm{SW}$ modes [revealed even better by injecting a dc current through the nanopillar; see Fig. 3(b)]. Thus, a small elliptical shape produced during the nanostructuration of the nanopillar is most likely responsible for the double peak observed at $H_{\mathbf{c}}$ in Fig. 2(b).

To summarize, the comparison between $3 \mathrm{D}$ simulations and experiments demonstrate that the observed mode splittings originate from a small asymmetry in the lateral confinement of the nanopillar.

\section{CONCLUSION}

In summary, we used the MRFM technique ${ }^{38}$ to study the SW eigenmodes in the prototype of a STNO, a normally magnetized nanopillar composed of two magnetic layers coupled by dipolar interaction.

In contrast to transport spectroscopy techniques, ${ }^{22,25}$ MRFM is sensitive to all SW modes excited in the sample ${ }^{39}$ and is completely independent of the transport properties of the studied spin-valve sample. Therefore, MRFM provides an alternative and complementary view on the magnetization dynamics in hybrid magnetic nanostructures. The additional advantages of the MRFM technique are its high sensitivity (in this study, it was able to detect angles of precession as low as $1^{\circ}$ in the thin magnetic layer) and its ability to operate on standard STNO devices buried under contact electrodes without a specific probe access to the studied sample. $^{43}$

Using MRFM, we were able to compare the SW spectra of a passive perpendicularly magnetized STNO-like sample excited by a uniform in-plane rf magnetic field and by an $\mathrm{rf}$ current flowing perpendicularly through the layers. We found that distinctly different $\mathrm{SW}$ modes (having azimuthal indices $\ell=0$ and $\ell=+1$, respectively) are excited by the two abovementioned excitation methods. By studying the influence of a spin-polarized dc current on the observed SW spectra we were able to determine which of the magnetic layers of the studied nanopillar plays the dominant role in the magnetization dynamics, resulting in the appearance of each particular SW mode.

We also developed a simple analytic theory making it possible to perform a comprehensive labeling of all the SW eigenmodes of a magnetic nanopillar in the studied axially symmetric case. This labeling requires three independent indices: the usual azimuthal and radial indices $\ell$ and $n$ used for the SW modes of a single magnetic disk and an additional index referring to the binding or antibinding ( $B$ or $A$ ) coupling between the two magnetic disks forming a nanopillar. The obtained experimental and analytic results were also compared to the results of 3D micromagnetic simulations obtained with the SpinFlow 3D package ${ }^{74}$ which confirmed the mode labeling obtained from the analytic theory.

Thus, we learned that in the axially symmetric case of a perpendicularly magnetized nanopillar, the excitations by the rf field and the rf current lead to two mutually orthogonal (and mutually exclusive) sets of excited SW modes: Only the $\ell=0$ modes are excited by the uniform rf magnetic field, while only the $\ell=+1$ modes are excited by the rf current. Therefore, the $\ell$ index, related to the azimuthal symmetry of the SW modes, is the discriminating parameter for the selection rules of the SW mode excitation.

Moreover, we have demonstrated experimentally and numerically that the mode selection rules are affected by the breaking of the axial symmetry of the studied nanopillar, either by tilting the bias magnetic field or by making the sample cross-section elliptical. In particular, if the axial symmetry is broken by tilting the bias magnetic field, the $\ell=0$ modes can also be excited by an rf current. This excitation is caused by the ST-FMR mechanism working when the magnetization vectors in the two magnetic layers of the nanopillar are not collinear. Also, the importance of the dynamic dipolar interaction between the magnetic layers of the nanopillar have been clearly demonstrated by our results. 
We believe that our results are important for the optimization of the characteristics of nanospintronic devices, and in particular STNOs, and for the experimental determination of the STNO parameters.

First of all, an accurate identification of the SW modes that can be excited in an STNO nanopillar is necessary to understand the details of the high-frequency STNO dynamics. The proposed identification of the nanopillar SW modes can be used for the experimental determination of the nanopillar characteristics, such as radius, static magnetization, gyromagnetic ratio, and dissipation. We note that in traditional STNO experiments, where the magnetization dynamics in a magnetic nanopillar is excited by a spin-polarized bias current creating a significant Oersted magnetic field with the $\ell=+1$ symmetry, it is easy to mix up the $\ell=0$ and the $\ell=+1$ SW eigenmodes. The spectra of these modes are in almost translational correspondence, and the experimentally observed dependence of the mode frequencies on the bias magnetic field can be well described by the traditional Kittel expression (see, for example, Ref. 11). Thus, the possibly excited $\ell=+1$ mode can be easily interpreted as a $\ell=0$ mode, which will lead to the apparent reduction of the "free" layer static magnetization necessary to fit the Kittel expression for the mode frequency. For instance, mislabeling the lowest energy mode of the $\ell=$ $+1 \mathrm{SW}$ spectrum as the uniform mode $(\ell, n=0,0)$ combined with a small misalignment of the applied field would lead to a discrepancy as large as $1 \mathrm{kG}$ in our case.

Second, the fact that in most cases both magnetic layers of a nanopillar take part in current-induced magnetization dynamics is very important for the correct identification of the excited $\mathrm{SW}$ modes. The collective (coupled) character of the SW modes in a nanopillar can directly influence the magnitude of the spin-transfer torque, which is dependent on the relative orientation of the magnetization vectors in the two magnetic layers. One might expect that the efficiency of the spin transfer torque for a particular SW mode depends not only on which layer ("free" or "fixed") is dominating the mode dynamics, but also on the coupling (in phase or antiphase) between the magnetization precession in two layers. In our experimental case, the interlayer coupling is in-phase for the SW modes dominated by the dynamics in a free (thin) layer. To obtain an interlayer coupling, which is antiphase for the SW modes dominated by the dynamics of the thin layer would require for example to increase its magnetization compared to the fixed layer one.

Finally, it is important to note that the MRFM technique has allowed us to study spin transfer effects in the axially symmetric configuration of a perpendicularly magnetized nanopillar, where the excitation of magnetization dynamics by ST-FMR vanishes due to the symmetry reasons. This geometry is rather important for applications as the excited SW modes have the maximum nonlinear frequency shift coefficient. ${ }^{19}$ This creates the maximum agility of the mode frequency with the bias current and, therefore, the maximum width of the synchronization band to the external periodic signal and to the large arrays of other STNOs. ${ }^{19}$ Phase synchronization has been identified as a possible mean to dramatically increase the generated microwave power of these nano-oscillators and, at the same time, reduce their linewidth. ${ }^{82-89}$

\section{ACKNOWLEDGMENTS}

This research was partially supported by the European Grant Master (NMP-FP7 212257) and by the French Grant Voice (ANR-09-NANO-006-01), by the contract from the US Army TARDEC, RDECOM, and by Grants No. ECCS1001815 and No. DMR-1015175 from the National Science Foundation of the United States.

\section{APPENDIX A: THEORETICAL MATERIAL}

\section{Equation of motion}

In this Appendix, we detail the derivation of the equations which govern the dynamics of a ferromagnetic layer in the presence of an external periodic excitation and of spin transfer, following the general formalism introduced in Sec. IV A 1. For an isolated layer, the local dynamics (within the exchange length) of the magnetization vector is described by the LandauLifshitz (LL) equation:

$$
\frac{1}{\gamma} \frac{\partial \boldsymbol{M}}{\partial t}=\boldsymbol{H} \times \boldsymbol{M}+\boldsymbol{h}(t) \times \boldsymbol{M},
$$

with $\gamma$ being the modulus of the gyromagnetic ratio. The LL equation is written here in its perturbative form, where the second term on the right-hand-side of Eq. (A1) represents the perturbation term. The field $\boldsymbol{H}$ is the effective magnetic field:

$$
\boldsymbol{H}=\boldsymbol{H}_{0}-4 \pi \widehat{\boldsymbol{G}} * \boldsymbol{M} .
$$

Here $\boldsymbol{H}_{0}$ is the total static external magnetic field (possibly spatially dependent) and the linear tensor self-adjoint operator $\widehat{G}$ describes the magnetic self-interactions.

Considering only the linear processes, we can represent the time-dependent (out-of-equilibrium) part of the magnetization as a series over the $\mathrm{SW}$ eigenmodes:

$$
\begin{aligned}
\boldsymbol{M}(t, \boldsymbol{r})-M_{s} \hat{\boldsymbol{u}}(\boldsymbol{r}) & \approx \boldsymbol{m}(t, \boldsymbol{r}) \\
& \approx \sum_{v} c_{v}(t) \boldsymbol{m}_{v}(\boldsymbol{r})+\text { c.c. }
\end{aligned}
$$

where $M_{s}$ is the saturation magnetization of the layer. Here, c.c. stands for the complex-conjugated part. The coefficients $c_{\nu}(t)$ are time-dependent SW amplitudes.

The second term on the left-hand-side of Eq. (A1) represents the perturbations from the equilibrium state, including the nonadiabatic contributions. The nonconservative perturbation magnetic field $\boldsymbol{h}(t)$ may depend on time and be a function of the magnetization distribution $\boldsymbol{M}(t)$. It can be approximately represented as

$$
\boldsymbol{h}(t)=\boldsymbol{h}_{1}(t)+\widehat{\boldsymbol{L}}_{1} * \boldsymbol{m}(t)
$$

where $\boldsymbol{h}_{1}(t)$ is the external perturbation field and $\widehat{\boldsymbol{L}}_{1}$ is a certain linear operator, allowing $\boldsymbol{h}(t)$ to depend on the magnetization distribution. The latter case may describe the influence of the Gilbert damping $-\left(\alpha / \gamma M_{s}\right) \partial \boldsymbol{M} / \partial t$ through

$$
\widehat{\boldsymbol{L}}_{1} * \boldsymbol{m}=i \frac{\alpha}{\gamma} \sum_{v} \omega_{v}\left[c_{v}(t) \boldsymbol{m}_{v}(\boldsymbol{r})-\text { c.c. }\right],
$$

where $\alpha$ is the Gilbert damping constant. 
Substituting the series representation Eq. (A3) and the representation of the perturbation field Eq. (A4) into Eq. (A1) and using orthogonality relations Eq. (7), one can obtain the following equations for the SW amplitudes $c_{\nu}$ :

$$
\frac{d c_{v}}{d t}=-i \omega_{\nu} c_{v}+i \gamma \sum_{v^{\prime}}\left(S_{v, v^{\prime}} c_{v^{\prime}}+S_{\nu, \bar{v}^{\prime}} \bar{c}_{v^{\prime}}\right)+i \gamma h_{\nu},
$$

where

$$
\begin{aligned}
S_{v, v^{\prime}} & =\frac{\left\langle\overline{\boldsymbol{m}}_{v} \cdot \widehat{\boldsymbol{L}}_{1} * \boldsymbol{m}_{v^{\prime}}\right\rangle-\left\langle\left(\hat{\boldsymbol{u}} \cdot \boldsymbol{h}_{1}\right)\left(\overline{\boldsymbol{m}}_{v} \cdot \boldsymbol{m}_{v^{\prime}}\right)\right\rangle}{\mathcal{N}_{v}}, \\
S_{v, \bar{v}^{\prime}} & =\frac{\left\langle\overline{\boldsymbol{m}}_{v} \cdot \widehat{\boldsymbol{L}}_{1} * \overline{\boldsymbol{m}}_{v^{\prime}}\right\rangle-\left\langle\left(\hat{\boldsymbol{u}} \cdot \boldsymbol{h}_{1}\right)\left(\overline{\boldsymbol{m}}_{v} \cdot \overline{\boldsymbol{m}}_{v^{\prime}}\right)\right\rangle}{\mathcal{N}_{v}}, \\
h_{v} & =\frac{\left\langle\overline{\boldsymbol{m}}_{v} \cdot \boldsymbol{h}_{1}\right\rangle}{\mathcal{N}_{v}} .
\end{aligned}
$$

In many cases the perturbed equations (A6) can be further simplified by retaining only the diagonal term $S_{v, v}=$ $i \alpha \omega_{v}\left\langle\overline{\boldsymbol{m}}_{v} \cdot \boldsymbol{m}_{v}\right\rangle /\left(\gamma \mathcal{N}_{v}\right)$ (assuming that there are no degenerate modes). The $\mathrm{SW}$ damping rate is then given by

$$
\Gamma_{v}=\alpha \omega_{v} \frac{\left\langle\overline{\boldsymbol{m}}_{v} \cdot \boldsymbol{m}_{v}\right\rangle}{\mathcal{N}_{v}} .
$$

The damping rate $\Gamma_{v}$ is responsible for the finite linewidth of the resonance peaks, $\Delta H$ (FWHM). If the sample is homogeneously magnetized and the precession is circular, the simple relation $\Delta H=2 \alpha \omega_{\nu} / \gamma$ holds.

From the equations above, one recovers for the coefficient $c_{v}$ the equation of motion of a damped harmonic oscillator:

$$
\frac{d c_{v}}{d t}=-i \omega_{\nu} c_{\nu}-\Gamma_{\nu} c_{\nu}+i \gamma h_{\nu}
$$

If a second magnetic layer $j^{\prime}$ is electrically connected to layer $j$ and spin transfer is allowed between them, the equation of motion must be modified. When a charge current $I$ is flowing through the layers, the additional Slonczewski-Berger term ${ }^{6,7}$ adds to the operator $\widehat{\boldsymbol{L}}_{1}$ of Eq. (A4). It is possible to define an effective damping in layer $j$, which depends on the spin-polarized current and on the relative angle between the magnetization in layer $j$ and the direction of the spin polarization $\hat{\boldsymbol{u}}_{j^{\prime}} \cdot{ }^{19}$

$$
\Gamma_{v}^{*}=\alpha \omega_{v} \frac{\left\langle\overline{\boldsymbol{m}}_{v} \cdot \boldsymbol{m}_{v}\right\rangle}{\mathcal{N}_{v}}+\frac{I \epsilon}{2 e N_{j}} \frac{\left\langle\left(\overline{\boldsymbol{m}}_{v} \cdot \boldsymbol{m}_{v}\right)\left(\hat{\boldsymbol{u}}_{j} \cdot \hat{\boldsymbol{u}}_{j^{\prime}}\right)\right\rangle}{\mathcal{N}_{v}},
$$

where

$$
N_{j}=\frac{M_{j} V_{j}}{\gamma \hbar}
$$

is the dimensionless total number of magnons that can be excited inside the volume $V_{j}$ of layer $j$. Here $\hbar$ is the reduced Planck constant, $e$ the modulus of the electron charge, and $\epsilon$ the spin polarization efficiency of the current. The threshold current for auto-oscillations in layer $j$ corresponds to $\Gamma_{v}^{*}=0$, that is, if $\hat{\boldsymbol{u}}_{j} \| \hat{\boldsymbol{u}}_{j}^{\prime}, I_{\mathrm{th}}=-2 \alpha \omega_{v} N_{j} e / \epsilon$. Using $\epsilon=0.3$ and the parameters of our thin layer, one can estimate $I_{\text {th }} \simeq-4.8 \mathrm{~mA}$ for the uniform SW mode at $8.1 \mathrm{GHz}$, in agreement with the experimental data. ${ }^{90}$ This result is also in quantitative agreement with calculations performed for our nanopillar device with no adjusting parameters in the framework of continuous random matrix theory (CRMT) described in Ref. 45.

We now turn to the periodic external excitation $\boldsymbol{h}_{1}(t)=$ $\boldsymbol{h}_{1} \exp ^{i \omega t}$, whose amplitude $\boldsymbol{h}_{1}$ is composed of three different contributions,

$$
\boldsymbol{h}_{1}=\boldsymbol{h}_{\mathrm{u}}+\boldsymbol{h}_{\mathrm{Oe}}+\boldsymbol{h}_{\mathrm{ST}},
$$

which we detail below.

The first type of excitation corresponds to a uniform rf magnetic field applied perpendicularly to the effective field $\boldsymbol{H}$. This configuration corresponds to conventional FMR spectroscopy. Assuming that the sample is uniformly magnetized along the nanopillar symmetry axis $\hat{z}$, it reduces to

$$
\boldsymbol{h}_{\mathrm{u}}=h_{\mathrm{rf}} \hat{\boldsymbol{x}}
$$

where $\hat{\boldsymbol{x}}$ is a unit vector in the in-plane direction and $h_{\mathrm{rf}}$ the linearly polarized amplitude.

SW spectroscopy can also be performed by injecting a uniform rf charge current $i_{\mathrm{rf}}$ through the nanopillar (i.e., along $\hat{z})$. First, this produces an orthoradial rf Oersted field:

$$
\boldsymbol{h}_{\mathrm{Oe}}=\left[\frac{4 \pi}{10}\right] \frac{i_{\mathrm{rf}}}{2 \pi R} \frac{\rho}{R}(-\sin \phi \hat{\boldsymbol{x}}+\cos \phi \hat{\boldsymbol{y}}),
$$

where $R$ is the radius of the nanopillar and $(\rho, \phi)$ are the polar coordinates. In this formula, the current should be expressed in $\mathrm{A}$ and the prefactor between the square brackets converts $\mathrm{A} / \mathrm{cm}$ into Oe (cgs units). The maximum amplitude of the rf Oersted field is reached at the periphery of the nanopillar, $\rho=R$, and equals 1.6 Oe for a peak amplitude $i_{\text {rf }}=100 \mu \mathrm{A}$ and the experimental parameters.

Second, the rf current produces a ST-FMR excitation:

$$
\boldsymbol{h}_{\mathrm{ST}}=\frac{i_{\mathrm{rf}}}{2 \pi \lambda}\left[\hat{\boldsymbol{u}}_{j} \times \hat{\boldsymbol{u}}_{j^{\prime}}\right] .
$$

where we have rewritten the spin-transfer efficiency of the charge current in Eq. (A10) as a function of

$$
2 \pi \lambda=\gamma \frac{2 e N_{j}}{\epsilon},
$$

which has the dimension of a distance $(\lambda \simeq 200 \mathrm{~nm}$ for our thin layer). If the thin and thick layers are misaligned by an angle $\beta$ in the plane $(y, z)$, Eq. (A15) reduces to $\boldsymbol{h}_{\mathrm{ST}}=i_{\mathrm{rf}} /(2 \pi \lambda) \sin \beta \hat{\boldsymbol{x}}$, which demonstrates that the ST-FMR excitation is equivalent to a linearly polarized $\mathrm{rf}$ magnetic field [Eq. (A13)]. The ST-FMR excitation vanishes if the magnetic layers are parallel. The amplitude ratio between the ST-FMR and the rf Oersted field excitations, both produced by the rf current flowing through the nanopillar, is $h_{\mathrm{ST}} / h_{\mathrm{Oe}} \simeq$ $(R / \lambda) \sin \beta$. In our geometry, $\lambda \approx 2 R$, but due to the small angle $\beta$ between the layers, the rf Oersted field contribution is much larger than the ST-FMR one. We note that even if $\beta \approx \pi / 2$, the contribution of the rf Oersted field cannot be disregarded in general in ST-FMR experiments.

\section{Numerical application}

In this section, we derive a practical guideline to calculate the eigenfrequencies $\omega_{v}$ using the analytical formalism developed in Sec. IV A. Let $\boldsymbol{m}_{v}$ be a certain orthogonal basis in the space of the vector functions $\boldsymbol{m}$ satisfying both the 
local orthogonality to $\hat{\boldsymbol{u}}$ and the total pinning condition at the boundary of the magnetic body. Spin-wave eigenmodes can thus be expressed as a series expansion on the $\boldsymbol{m}_{v}$ basis [cf. Eq. (A3)]. A general expression for the eigenfrequencies can be found from the condition of vanishing determinant,

$$
\left\|\mathcal{N}_{v^{\prime}, v} \omega-\mathcal{N}_{v^{\prime}, v}\{\widehat{\boldsymbol{\Omega}}\}_{v^{\prime}, v}\right\|=0,
$$

where for the simplicity of the discussion, it is convenient to introduce a curly bracket notation, to indicate that the enclosed quantity is spatially weighted by the spatial pattern of the mode profile and averaged:

$$
\{\widehat{\boldsymbol{\Omega}}\}_{v^{\prime}, v} \equiv \frac{\left\langle\overline{\boldsymbol{m}}_{v^{\prime}} \cdot \widehat{\boldsymbol{\Omega}} * \boldsymbol{m}_{v}\right\rangle}{\mathcal{N}_{v^{\prime}, v}} .
$$

This echoes the chevron-bracket notation introduced in Eq. (1) to indicate the homogeneous spatial average over the volume of the magnetic body. Here $\mathcal{N}$ represents a renormalization quantity, defined by

$$
\mathcal{N}_{v^{\prime}, v} \equiv i\left\langle\overline{\boldsymbol{m}}_{v^{\prime}} \cdot\left(\hat{\boldsymbol{u}} \times \boldsymbol{m}_{v}\right)\right\rangle,
$$

which has in general off-diagonal elements.

In the case of perpendicularly magnetized disks, where the set of Bessel functions $\frac{1}{2}(\hat{\boldsymbol{x}}+i \hat{\boldsymbol{y}}) e^{-i \ell \phi} \boldsymbol{J}_{\ell}\left(k_{\ell, n} \rho\right)$ diagonalizes the uncoupled Hamiltonian, the secular Eq. (A17) becomes diagonal and we recover Eq. (8):

$$
\omega_{v}=\{\widehat{\boldsymbol{\Omega}}\}_{v, v} .
$$

We now perform the numerical application of the eigenvalue of the lowest energy mode $(\ell, n=0,0)$ using the parameters of our nanopillar shown in Table I. We drop the subscript $v$ to the curly brackets, understanding that the spatial average in Eq. (A20) is made over the uniform mode $\boldsymbol{m}_{v}=\frac{1}{2} J_{0}\left(k_{0} \rho\right)(\hat{\boldsymbol{x}}+i \hat{\boldsymbol{y}})$, where $k_{0}=2.4048 / R$ is its wave vector. In this case, the value of the normalization constant is simply $\mathcal{N}_{0}=\left\langle J_{0}^{2}\right\rangle=J_{1}^{2}\left(k_{0} R\right)=0.2695$.

The different contributions that enter inside the operator $\{\widehat{\boldsymbol{\Omega}}\}$ are detailed in Eq. (4):

$$
\{\widehat{\boldsymbol{\Omega}}\}=\gamma\{H\}+4 \pi \gamma M_{j}\{\widehat{\boldsymbol{G}}\} .
$$

We start with the calculation of the amplitude of effective magnetic field, the first term on the right-hand side of Eq. (A21). As shown by Eq. (5), the scalar value $H$ along $\hat{z}$ decomposes itself in two terms:

$$
\{H\}=\left\{\hat{z} \cdot \boldsymbol{H}_{0}\right\}-4 \pi M_{s}\{\hat{z} \cdot \widehat{\boldsymbol{G}} * \hat{z}\} .
$$

The term $\hat{z} \cdot \widehat{G} * \hat{z}$ represents the static magnetic selfinteraction. In the case of homogeneously magnetized body, the inhomogeneous exchange contribution to the static selfinteraction is strictly null and the second term of Eq. (A22) reduces to the magnetodipolar contribution $\widehat{\boldsymbol{G}}^{(d)}$, which has the following form in the wave-vector representation:

$$
\widehat{\boldsymbol{G}}^{(d)}(\boldsymbol{r})=\int D(\boldsymbol{k}) \frac{\boldsymbol{k} \otimes \boldsymbol{k}}{k^{2}} \exp ^{i \boldsymbol{k} \cdot \boldsymbol{r}} d^{3} \boldsymbol{k},
$$

where $D(\boldsymbol{k})$ is the Fourier transform of the body shape function $^{91}$ and the symbol $\otimes$ denotes direct product of vectors. For a disk of radius $R$ and thickness $t$, an analytical expression for the different position-dependent demagnetization tensor
TABLE III. Values of the self- and cross- depolarization tensor elements weighted by the precession profile of the uniform mode for the thin $(j=a)$ and thick $(j=b)$ disks.

\begin{tabular}{lcccc}
\hline \hline & $(a, a)$ & $(a, b)$ & $(b, a)$ & $(b, b)$ \\
\hline$\left\{N_{z z}^{\left(j, j^{\prime}\right)}\right\}$ & +0.979 & -0.068 & -0.017 & +0.919 \\
$\left\{N_{x x}^{\left(j, j^{\prime}\right)}\right\}$ & +0.016 & +0.042 & +0.011 & +0.056 \\
\hline \hline
\end{tabular}

elements of a disk $N_{u v[R, t]}(\boldsymbol{r}) \equiv \hat{\boldsymbol{u}}(\boldsymbol{r}) \cdot \widehat{\boldsymbol{G}}^{(d)} * \hat{\boldsymbol{v}}$ valid in the whole space are available in Ref. 92. For perpendicularly magnetized disks where $\hat{\boldsymbol{u}}=\hat{\boldsymbol{v}}=\hat{\boldsymbol{z}}$, the expression of the self-integral becomes

$$
\left\{N_{z z}^{(j, j)}\right\}=\frac{1}{\left\langle J_{0}^{2}\right\rangle} \int_{V_{j}} d^{2} \rho d z J_{0}^{2}\left(k_{0} \rho\right) N_{z z\left[R, t_{j}\right]}(\rho, z)
$$

for both the thin $(j=a)$ and thick $(j=b)$ layers. Their numerical values are displayed in Table III.

The term $\left\{\hat{\boldsymbol{z}} \cdot \boldsymbol{H}_{0}\right\}$ of Eq. (A22) is the projection on the precession axis of the total applied magnetic field. It comprises the external magnetic field $\left\{H_{\text {ext }}\right\}=H_{\text {ext }}$, the stray field of the mechanical-FMR probe $\left\{H_{\text {sph }}\right\}=190$ Oe and the cross-magneto-dipolar static interactions between each layer. The latter can be estimated from the cross-tensor elements of the static magnetodipolar field of the $j^{\prime}$ th disk produced over the volume of the $j$ th disks:

$\left\{N_{z z}^{\left(j, j^{\prime}\right)}\right\}=\frac{1}{\left\langle J_{0}^{2}\right\rangle} \int_{V_{j}} d^{2} \rho d z J_{0}^{2}\left(k_{0} \rho\right) N_{z z\left[R, t_{j^{\prime}}\right]}\left(\rho, z+z_{0}\right)$,

where $z_{0}$ is the distance between the centers of the two axially aligned disks. The numerical values of the cross-tensor elements are reported in Table III. Putting all the above elements together, the total effective field simply writes

$$
\{H\}=H_{\mathrm{ext}}+\left\{H_{\mathrm{sph}}\right\}-4 \pi\left\{N_{z z}^{(j, j)}\right\} M_{j}-4 \pi\left\{N_{z z}^{\left(j, j^{\prime}\right)}\right\} M_{j^{\prime}} .
$$

We now turn our attention to the integration $4 \pi M_{j}\{\widehat{\boldsymbol{G}}\}$, the second term on the right-hand side of Eq. (A21). We recall that for Py the operator $\widehat{\boldsymbol{G}}=\widehat{\boldsymbol{G}}^{(e)}+\widehat{\boldsymbol{G}}^{(d)}$ is the sum of the inhomogeneous exchange and magnetodipolar interactions. In the wave-vector representation, $\widehat{\boldsymbol{G}}^{(e)}=\Lambda_{\mathrm{ex}}^{2} k^{2} \widehat{\boldsymbol{I}}$, where the exchange length $\Lambda_{\mathrm{ex}}=\sqrt{2 J /\left(4 \pi M_{j}^{2}\right)}$ depends on the exchange stiffness constant $J$, expressed in $\mathrm{erg} / \mathrm{cm}\left(=10^{-6}\right.$ in Py). It produces the exchange field

$$
\left\{H_{\mathrm{ex}}\right\}=4 \pi M_{j} \Lambda_{\mathrm{ex}}^{2} k_{0}^{2},
$$

which yields the value $\left\{H_{\mathrm{ex}}\right\}=110 \mathrm{Oe}$.

The other contribution is the dynamic magnetodipolar selfinteraction, which represents the depolarization field of the SW mode on itself. For the $\ell=0$ modes, an analytical expression 
can be derived:

$$
\begin{aligned}
\left\{N_{x x}^{\left(j, j^{\prime}\right)}\right\}= & \frac{1}{\left\langle J_{0}^{2}\right\rangle} \int_{V_{j}} d^{2} \rho d z J_{0}\left(k_{0} \rho\right) \\
& \times \int_{0}^{R} d u \frac{\partial N_{x x\left[u, t_{j^{\prime}}\right]}(\rho, z)}{\partial u} J_{0}\left(k_{0} u\right),
\end{aligned}
$$

where the quantity in the second integral is the magnetic stray field produced at the spatial position $\boldsymbol{r}$ by a cylindrical tube of width $d u$, radius $u$, and thickness $t_{j^{\prime}}$, homogeneously magnetized $^{93}$ along $\hat{\boldsymbol{x}}$ by $J_{0}\left(k_{0} u\right)$. We use the same expression above to write the self- and cross-contribution, understanding implicitly that the spacer value $z_{0}$ should be added in the later case, as shown in Eq. (A25). The values of the self- and cross-tensor elements are reported in the last line of Table III. We mention that an approximate expression of Eq. (A28) has been derived by Kalinikos and Slavin ${ }^{94}$ for the lowest SW branch of platelet shape bodies with uniform magnetization across the film thickness. This expression reduces to

$$
\left\{N_{x x}\right\} \simeq \frac{1}{2}\left(1-G_{0}^{\perp}\right),
$$

where the analytical expression of $G_{\ell, n}^{\perp}$ for Bessel functions is given by Eq. (26) in Ref. 38.

The cross elements are responsible for the dynamic dipolar coupling detailed in Sec. IV B:

$$
h_{j, j^{\prime}}=4 \pi\left\{N_{x x}^{\left(j, j^{\prime}\right)}\right\} M_{j^{\prime}} .
$$

The value of the coupling frequency $\Omega$ for the lowest energy mode yields

$$
\Omega \simeq \gamma \sqrt{\left\{N_{x x}^{(a, b)}\right\} 4 \pi M_{b}\left\{N_{x x}^{(b, a)}\right\} 4 \pi M_{a}},
$$

which leads to $\Omega / 2 \pi \simeq 0.56 \mathrm{GHz}$.

Neglecting the dynamical dipolar coupling [the generalization to $\Omega \neq 0$ is Eq. (15)], we derive an expression for the eigenvalue of index $v=j_{0,0}$ :

$$
\frac{\omega_{v}}{\gamma}=\{H\}+4 \pi\left\{N_{x x}^{(j, j)}\right\} M_{j}+\left\{H_{\mathrm{ex}}\right\},
$$

$j=a, b$ being the layer index and $\{H\}$ being defined in Eq. (A26). Equation (A32) is a simplified expression valid for circularly polarized modes $(\ell=0$ index $)$, where we have taken advantage of the equality $\left\{N_{x x}\right\}_{0}=\left\{N_{y y}\right\}_{0}$ in our circular disk. This expression can be extended to higher-order modes by using $\left\{N_{x x}\right\}_{\ell, n} \approx\left\{N_{x x}\right\}_{0} \sqrt{k_{\ell, n} / k_{0}}$ inside Eq. (A32). This approximation is derived from the ellipticity of $\ell \neq 0$ modes $\left(\left\{N_{x x}\right\} \neq\left\{N_{y y}\right\}\right)$. One needs thus two separate equations (A32) for the values of $\omega$ for each cartesian axis: ${ }^{38}$ one proportional to $m_{x}^{2} /\left(m_{x} m_{y}\right)$, the other to $m_{y}^{2} /\left(m_{x} m_{y}\right)$. The product of these two equations is independent of the ellipticity, leading to the general expression for the eigenvalue of arbitrary index $v=j_{\ell, n}$ :

$$
\begin{aligned}
\frac{\omega_{v}^{2}}{\gamma^{2}}= & \left(\{H\}_{v}+4 \pi\left\{N_{x x}^{(j, j)}\right\}_{v} M_{j}+\left\{H_{\mathrm{ex}}\right\}_{v}\right) \\
& \times\left(\{H\}_{v}+4 \pi\left\{N_{y y}^{(j, j)}\right\}_{v} M_{j}+\left\{H_{\mathrm{ex}}\right\}_{v}\right),
\end{aligned}
$$

which can be seen as a generalization of the Kittel formula for an arbitrarily shaped multibody.
Equating $H_{a_{00}}=H_{(3)}$ and $H_{b_{00}}=H_{(1}$ in Eq. (A32), where $H_{a_{00}}$ and $H_{b_{00}}$ are the resonance fields at $f_{\text {fix }}=8.1 \mathrm{GHz}$ of the uniform modes in the thin and thick disks, respectively, leads to $4 \pi M_{a}=8.0 \times 10^{3} \mathrm{G}$ and $4 \pi M_{b}=9.6 \times 10^{3} \mathrm{G}$.

Finally, the above formalism also makes it possible to determine the angle $\theta_{j}=\left(\hat{\boldsymbol{z}}, \hat{\boldsymbol{u}}_{j}\right)$ between the equilibrium direction of the magnetization in layer $j$ and the normal axis when the bias field is applied at a polar angle $\theta_{H}=\left(\hat{z}, \boldsymbol{H}_{\text {ext }}\right)$. From the equality $\hat{\boldsymbol{u}} \times \boldsymbol{H}_{\mathrm{ext}}=4 \pi M_{j} \hat{\boldsymbol{u}} \times\left\langle\widehat{\boldsymbol{G}}^{\left(d_{j}\right)} * \hat{\boldsymbol{u}}\right\rangle$, one extracts the relationship

$$
H_{\mathrm{ext}} \sin \left(\theta_{j}-\theta_{H}\right)=2 \pi M_{j}\left(\left\langle N_{z z}^{(j j)}\right\rangle-\left\langle N_{x x}^{(j j)}\right\rangle\right) \sin 2 \theta_{j} .
$$

The angle $\theta_{j}$ is useful to estimate the shift to lower field of the FMR spectrum, ${ }^{38} 2 \pi M_{j}\left(\left\{N_{z z}^{(j)}\right\}-\left\{N_{x x}^{(j)}\right\}\right)\left(1-\cos 2 \theta_{j}\right) \approx 420$ Oe, when $\theta_{b}=13^{\circ}$ in the thick layer.

\section{APPENDIX B: METHODS AND CALIBRATION}

\section{Mechanical vibration amplitude}

Here we detail the experimental protocol used to calibrate the amplitudes of the uniform rf magnetic field and of the mechanical-FMR signal. The procedure uses the nonlinear properties of the magnetization dynamics and consists of studying the power dependence of the line shape. In the following, $\Delta H$ denotes the FWHM linewidth measured in the linear regime.

We use the onset of foldover as a mean to calibrate the strength of the rf field produced by the microwave antenna. This nonlinear effect is responsible for the asymmetric shape of the resonance peaks in Figs. 2 and 3. In fact, it was pointed out by Anderson and Suhl ${ }^{52}$ that the resonance curve at high power should be skewed, due to the static change of the magnetization $M_{z}$, which also shifts the resonance frequency. For a normally magnetized sample, this nonlinear frequency shift is positive (blueshift), and the field-sweep line shapes are distorted toward low field. There is a critical strength of the rf magnetic field $h_{c}$ (linearly polarized amplitude) for which the slope of the resonance curve becomes infinite on the low field side of the resonance: ${ }^{53}$

$$
h_{c}=2 \Delta H \sqrt{\frac{2 \Delta H}{3 \sqrt{3}\left|\left\{N_{z z}\right\}-\left\{N_{x x}\right\}\right| 4 \pi M_{s}}},
$$

where $\left\{N_{z z}\right\}-\left\{N_{x x}\right\}$ is the difference between the depolarization factors in the longitudinal and transverse directions. Experimentally, we find that for the peak at $H_{\oplus}$, this onset is reached when the output power of the synthesizer at $8.1 \mathrm{GHz}$ is $P_{0}=+9 \mathrm{dBm}$. Using the magnetic properties of the thick layer (Table I), we infer from Eq. (B1) that at the critical onset of foldover, the strength of the rf magnetic field is $h_{c}=4.2 \pm 0.8 \mathrm{Oe}$. We note that this value is in agreement with the estimation made by directly evaluating the field produced by the rf current flowing in the antenna at $8.1 \mathrm{GHz}$ for this output power, $h_{\mathrm{rf}}=5.5 \pm 1$ Oe.

Furthermore, this procedure gives a calibration of the amplitude of the mechanical-FMR signal $\left\langle\Delta M_{z}\right\rangle$. At the onset 
of foldover, the longitudinal change of the magnetization is indeed $^{52}$

$$
4 \pi\left\langle\Delta M_{z}\right\rangle=\frac{4}{3 \sqrt{3}} \Delta H .
$$

A numerical application of Eq. (B2) yields $4 \pi\left\langle\Delta M_{z}\right\rangle=$ $36 \pm 4 \mathrm{G}$, which corresponds to the critical angle of precession $\left\langle\theta_{c}\right\rangle=5^{\circ}$. We have used this calibration of the cantilever vibration amplitude to evaluate the change of the longitudinal magnetization at the maximum of the peak at $H_{(1}$ in Fig. 2(a).

\section{Microwave setup}

In this Appendix, we give some details on the microwave circuit, which was carefully designed to minimize the crosstalk between the rf field and rf current excitation parts.

The calibration of the $\mathrm{rf}$ magnetic field produced by the microwave antenna has been presented in the previous Appendix. In order to calibrate the rf current flowing through the nanopillar with respect to the synthesizer output power injected into the contact electrodes, we have first used a standard microwave setup. The nanopillar electrodes are directly connected to the microwave synthesizer through a picoprobe, a bias-T, and a semirigid coaxial line, which make it possible to perform voltage-FMR spectroscopy. In this experiment, the amplitude of $i_{\text {rf }}$ flowing through the nanopillar can be accurately determined, owing to the determination of losses and reflections in the microwave circuit using a network analyzer. Then the same experiment is repeated inside the MRFM setup, in which the contact electrodes are wire bounded to a microwave cable and the circuit contains more connections. The comparison with the standard setup yields an estimation of the rms amplitude of the rf current in the mechanical-FMR setup: $i_{\mathrm{rf}}=170 \pm 40 \mu \mathrm{A}$ for an output power of $-22 \mathrm{dBm}$ injected at $8.1 \mathrm{GHz}$ through the contact electrodes.

It is also possible to estimate experimentally the highfrequency coupling between the microwave antenna and the electrodes that contact the nanopillar. For this, we exploit the fact that in the exact perpendicular configuration, different SW modes are excited by the uniform rf field $(\ell=0$ index $)$ and by the rf current $(\ell=+1$ index $)$. If the rf magnetic field used to excite the conventional FMR spectrum would induce any relevant $\mathrm{rf}$ eddy current through the nanopillar, $\ell=+1$ modes which are excited in the SW spectrum of Fig. 2(b) should also be detected in the SW spectrum of Fig. 2(a), which is not the case. We deduce from this observation that for an output power of $+3 \mathrm{dBm}$ injected in the antenna, the induced eddy current through the nanopillar is less than when injecting $-38 \mathrm{dBm}$ directly through the contact electrodes, that is, $i_{\text {rf }}<30 \mu \mathrm{A}$. So, at $f_{\text {fix }}=8.1 \mathrm{GHz}$, the isolation between the two parts of the microwave circuit is better than $40 \mathrm{~dB}$. However, we note that the latter depends on the frequency, and that for some particular values, it can drop to only $20 \mathrm{~dB}$.

Still, owing to the broadband design of the contact electrodes and to the low microwave power required to excite SW modes with the rf current excitation part, it is possible to acquire FMR spectra at a fixed-bias magnetic field $H_{\text {fix }}$ by sweeping the frequency of the rf current. We mention that in the frequency-sweep experiments presented in Fig. 4(a), the output power of the synthesizer is kept at $-22 \mathrm{dBm}$ over the full frequency range (4 to $18 \mathrm{GHz}$ ), which results in an amplitude variation of $i_{\mathrm{rf}}$, mainly associated with frequencydependent losses in the circuit. We also note that the same frequency-sweep experiment cannot be performed as cleanly with the rf field excitation due to the high power that has to be injected in the microwave antenna and to the dependence of the isolation on frequency mentioned above.

\section{Cavity-FMR characterization of the extended film}

Before the nanofabrication of the nanopillar devices, a reference film of $\mathrm{Cu} 60\left|\mathrm{Py}_{b} 15\right| \mathrm{Cu} 10\left|\mathrm{Py}_{a} 4\right| \mathrm{Au} 25$ (thicknesses in $\mathrm{nm}$ ) is cut out from the Si wafer for characterization purposes. The extraction of the material parameters is obtained independently on this reference film by a reflection X-band spectrometer $(9.6 \mathrm{GHz})$ operating at room temperature. The experiment consists of measuring the resonance spectra of the multilayer as a function of the polar angle $\theta_{H}$ between the applied field and the normal to the film. The resonance field of the layer $j=a, b$ as a function of $\theta_{H}$ depends only on the gyromagnetic ratio $\gamma$ and on the total perpendicular anisotropy field, which here reflects entirely the demagnetizing field $4 \pi M_{j}$ of the layer. ${ }^{95}$ The obtained values for the gyromagnetic ratio (identical for both layers) and the magnetizations are collected in Table I. The magnetization of the thick layer (9.6 kOe) corresponds to the expected value for bulk Py with composition $\mathrm{Ni}_{80} \mathrm{Fe}_{20}$. The magnetization of the thin layer is $1.4 \mathrm{kG}$ smaller, which reflects the reduction of the magnetization in the interfacial layer (of the order of $1 \mathrm{~nm}$ ), due to the gradual composition variation of the $\mathrm{NiFe}$ alloy from $\mathrm{Ni}_{80} \mathrm{Fe}_{20}$ to the normal metal $(\mathrm{Cu}$ or $\mathrm{Au}){ }^{59,61}$

An estimate of the damping parameter and the amount of inhomogeneous broadening can also be obtained from the angular dependence of the linewidth of the resonant mode associated with each layer. The linewidth is, in general, the sum of two contributions: an intrinsic relaxation of the magnetization vector (homogeneous width) and an inhomogeneous broadening corresponding to a distribution of resonance fields (whose main sources have been described for a permalloy polycrystalline layer ${ }^{95}$ ). The intrinsic damping parameter is deduced from the parallel geometry linewidth. In the $\mathrm{Py}_{b}$ 15-nm-thick layer, the linewidths observed in the parallel and perpendicular geometries are, respectively, $\Delta H_{\| b}=64$ Oe and $\Delta H_{\perp b}=73$ Oe. The higher value observed in the perpendicular geometry reveals a fair amount of inhomogeneities. ${ }^{95}$ The linewidth observed in the parallel geometry corresponds to an intrinsic damping parameter $\alpha_{b}=(0.9 \pm 0.1) \times 10^{-2}$. For the thin $\mathrm{Py}_{a}$ 4-nm layer, $\Delta H_{\| a}=83$ Oe and $\Delta H_{\perp a}=171$ Oe. From the parallel geometry linewidth, we deduce an intrinsic damping parameter $\alpha_{a}=(1.5 \pm 0.3) \times 10^{-2}$. This value is higher than for the thick layer because of the larger effect of the diffusion of the microwave magnetization of the conduction electrons in the adjacent normal metal layers, ${ }^{61}$ associated with the fact that the thin layer thickness $(4 \mathrm{~nm})$ is less than the spin-diffusion length in Py. The much larger value $\Delta H_{\perp a}$ is associated with a large contribution of the inhomogeneous broadening arising from a substantial effect of interfacial roughness (and dispersion of heights of the crystallites of the base) on the thin Py layer grown on top of a 85-nm-thick metallic base. 
*oklein@cea.fr

${ }^{1}$ S. Wolf, D. D. Awschalom, R. A. Buhrman, J. Daughton, S. von Molnar, M. L. Roukes, A. Y. Chtchelkanova, and D. M. Treger, Science 294, 1488 (2001).

${ }^{2}$ M. N. Baibich, J. M. Broto, A. Fert, F. Nguyen Van Dau, F. Petroff, P. Etienne, G. Creuzet, A. Friederich, and J. Chazelas, Phys. Rev. Lett. 61, 2472 (1988).

${ }^{3}$ G. Binasch, P. Grunberg, F. Saurenbach, and W. Zinn, Phys. Rev. B 39, 4828 (1989).

${ }^{4}$ B. Dieny, V. Speriosu, B. Gurney, S. Parkin, D. Wilhoit, K. Roche, S. Metin, D. Peterson, and S. Nadimi, J. Magn. Magn. Mater. 93, 101 (1991).

${ }^{5}$ M. Pannetier, C. Fermon, G. L. Goff, J. Simola, and E. Kerr, Science 304, 1648 (2004).

${ }^{6}$ J. Slonczewski, J. Magn. Magn. Mater. 159, L1 (1996).

${ }^{7}$ L. Berger, Phys. Rev. B 54, 9353 (1996).

${ }^{8}$ M. Tsoi, A. G. M. Jansen, J. Bass, W.-C. Chiang, M. Seck, V. Tsoi, and P. Wyder, Phys. Rev. Lett. 80, 4281 (1998).

${ }^{9}$ F. J. Albert, J. A. Katine, R. A. Buhrman, and D. C. Ralph, Appl. Phys. Lett. 77, 3809 (2000).

${ }^{10}$ J. Grollier, V. Cros, A. Hamzic, J. M. George, H. Jaffres, A. Fert, G. Faini, J. Ben Youssef, and H. Legall, Appl. Phys. Lett. 78, 3663 (2001).

${ }^{11}$ S. I. Kiselev, J. C. Sankey, I. N. Krivorotov, N. C. Emley, R. J. Schoelkopf, R. A. Buhrman, and D. C. Ralph, Nature (London) 425, 380 (2003).

${ }^{12}$ W. H. Rippard, M. R. Pufall, S. Kaka, S. E. Russek, and T. J. Silva, Phys. Rev. Lett. 92, 027201 (2004).

${ }^{13}$ V. E. Demidov, S. O. Demokritov, B. Hillebrands, M. Laufenberg, and P. P. Freitas, Appl. Phys. Lett. 85, 2866 (2004).

${ }^{14}$ G. Woltersdorf, O. Mosendz, B. Heinrich, and C. H. Back, Phys. Rev. Lett. 99, 246603 (2007).

${ }^{15}$ G. de Loubens, V. V. Naletov, O. Klein, J. B. Youssef, F. Boust, and N. Vukadinovic, Phys. Rev. Lett. 98, 127601 (2007).

${ }^{16}$ G. de Loubens, V. V. Naletov, M. Viret, O. Klein, H. Hurdequint, J. Ben Youssef, F. Boust, and N. Vukadinovic, J. Appl. Phys. 101, 09F514 (2007).

${ }^{17}$ G. Gubbiotti, M. Madami, S. Tacchi, G. Carlotti, H. Tanigawa, and T. Ono, J. Phys. D: Appl. Phys. 41, 134023 (2008).

${ }^{18}$ P. S. Keatley, V. V. Kruglyak, A. Neudert, E. A. Galaktionov, R. J. Hicken, J. R. Childress, and J. A. Katine, Phys. Rev. B 78, 214412 (2008).

${ }^{19}$ A. Slavin and V. Tiberkevich, IEEE Trans. Magn. 45, 1875 (2009).

${ }^{20}$ A. V. Nazarov, H. S. Cho, J. Nowak, S. Stokes, and N. Tabat, Appl. Phys. Lett. 81, 4559 (2002).

${ }^{21}$ N. Stutzke, S. L. Burkett, and S. E. Russek, Appl. Phys. Lett. 82, 91 (2003).

${ }^{22}$ S. Petit, C. Baraduc, C. Thirion, U. Ebels, Y. Liu, M. Li, P. Wang, and B. Dieny, Phys. Rev. Lett. 98, 077203 (2007).

${ }^{23}$ A. Helmer, S. Cornelissen, T. Devolder, J.-V. Kim, W. van Roy, L. Lagae, and C. Chappert, Phys. Rev. B 81, 094416 (2010).

${ }^{24}$ A. A. Tulapurkar, Y. Suzuki, A. Fukushima, H. Kubota, H. Maehara, K. Tsunekawa, D. D. Djayaprawira, N. Watanabe, and S. Yuasa, Nature (London) 438, 339 (2005).

${ }^{25}$ J. C. Sankey, P. M. Braganca, A. G. F. Garcia, I. N. Krivorotov, R. A. Buhrman, and D. C. Ralph, Phys. Rev. Lett. 96, 227601 (2006).

${ }^{26}$ W. Chen, J.-M. L. Beaujour, G. de Loubens, A. D. Kent, and J. Z. Sun, Appl. Phys. Lett. 92, 012507 (2008).

${ }^{27}$ W. Chen, G. de Loubens, J.-M. L. Beaujour, A. D. Kent, and J. Z. Sun, J. Appl. Phys. 103, 07 A502 (2008).
${ }^{28}$ N. Biziere, E. Murè, and J.-P. Ansermet, Phys. Rev. B 79, 012404 (2009).

${ }^{29}$ C. T. Boone, J. A. Katine, J. R. Childress, V. Tiberkevich, A. Slavin, J. Zhu, X. Cheng, and I. N. Krivorotov, Phys. Rev. Lett. 103, 167601 (2009).

${ }^{30}$ W. H. Rippard, A. M. Deac, M. R. Pufall, J. M. Shaw, M. W. Keller, S. E. Russek, G. E. W. Bauer, and C. Serpico, Phys. Rev. B 81, 014426 (2010).

${ }^{31}$ N. Biziere and C. Fermon, Appl. Phys. Lett. 92, 092503 (2008).

${ }^{32}$ N. Biziere and C. Fermon, Phys. Rev. B 78, 064408 (2008).

${ }^{33}$ D. Houssameddine, U. Ebels, B. Delaët, B. Rodmacq, I. Firastrau, F. Ponthenier, M. Brunet, C. Thirion, J.-P. Michel, L. PrejbeanuBuda, M.-C. Cyrille, O. Redon, and B. Dieny, Nat. Mater. 6, 447 (2007).

${ }^{34}$ Z. Zhang, P. C. Hammel, and P. E. Wigen, Appl. Phys. Lett. 68, 2005 (1996).

${ }^{35}$ K. Wago, D. Botkin, C. S. Yannoni, and D. Rugar, Appl. Phys. Lett. 72, 2757 (1998).

${ }^{36}$ A. Jander, J. Moreland, and P. Kabos, J. Appl. Phys. 89, 7086 (2001).

${ }^{37}$ V. Charbois, V. V. Naletov, J. Ben Youssef, and O. Klein, J. Appl. Phys. 91, 7337 (2002).

${ }^{38}$ O. Klein, G. de Loubens, V. V. Naletov, F. Boust, T. Guillet, H. Hurdequint, A. Leksikov, A. N. Slavin, V. S. Tiberkevich, and N. Vukadinovic, Phys. Rev. B 78, 144410 (2008).

${ }^{39}$ G. de Loubens, V. V. Naletov, and O. Klein, Phys. Rev. B 71, 180411 (2005).

${ }^{40}$ V. V. Naletov, G. de Loubens, V. Charbois, O. Klein, V. S. Tiberkevich, and A. N. Slavin, Phys. Rev. B 75, 140405 (2007).

${ }^{41}$ G. de Loubens, A. Riegler, B. Pigeau, F. Lochner, F. Boust, K. Y. Guslienko, H. Hurdequint, L. W. Molenkamp, G. Schmidt, A. N. Slavin, V. S. Tiberkevich, N. Vukadinovic, and O. Klein, Phys. Rev. Lett. 102, 177602 (2009).

${ }^{42}$ B. Pigeau, G. de Loubens, O. Klein, A. Riegler, F. Lochner, G. Schmidt, L. W. Molenkamp, V. S. Tiberkevich, and A. N. Slavin, Appl. Phys. Lett. 96, 132506 (2010).

${ }^{43}$ B. Pigeau, G. de Loubens, O. Klein, A. Riegler, F. Lochner, G. Schmidt, and L. W. Molenkamp, Nat. Phys. 7, 26 (2011).

${ }^{44} \mathrm{We}$ mention here that the measured amplitude of the GMR effect in our sample is $\Delta R_{\mathrm{GMR}}=25 \mathrm{~m} \Omega$, in agreement with the value calculated by CRMT for the multilayer stack composition. ${ }^{45}$

${ }^{45}$ V. S. Rychkov, S. Borlenghi, H. Jaffres, A. Fert, and X. Waintal, Phys. Rev. Lett. 103, 066602 (2009).

${ }^{46}$ O. Klein, V. Charbois, V. V. Naletov, and C. Fermon, Phys. Rev. B 67, 220407(R) (2003).

${ }^{47}$ O. Klein, V. Charbois, V. V. Naletov, and C. Fermon, J. Magn. Magn. Mater. 272, E1027 (2004).

${ }^{48}$ I. Lee, Y. Obukhov, G. Xiang, A. Hauser, F. Yang, P. Banerjee, D. Pelekhov, and P. Hammel, Nature (Lodnon) 466, 845 (2010).

${ }^{49}$ V. Charbois, V. V. Naletov, J. Ben Youssef, and O. Klein, Appl. Phys. Lett. 80, 4795 (2002).

${ }^{50}$ V. V. Naletov, V. Charbois, O. Klein, and C. Fermon, Appl. Phys. Lett. 83, 3132 (2003).

${ }^{51}$ R. E. Arias and D. L. Mills, Phys. Rev. B 79, 144404 (2009).

${ }^{52}$ P. W. Anderson and H. Suhl, Phys. Rev. 100, 1788 (1955).

${ }^{53}$ E. Schlömann, Technical Report No. R-48, 1959 (unpublished).

${ }^{54}$ W. Chen, G. de Loubens, J.-M. L. Beaujour, J. Z. Sun, and A. D. Kent, Appl. Phys. Lett. 95, 172513 (2009). 
${ }^{55}$ Y. Tserkovnyak, A. Brataas, G. E. W. Bauer, and B. I. Halperin, Rev. Mod. Phys. 77, 1375 (2005).

${ }^{56}$ M. V. Costache, M. Sladkov, S. M. Watts, C. H. van der Wal, and B. J. van Wees, Phys. Rev. Lett. 97, 216603 (2006).

${ }^{57}$ J. N. Kupferschmidt, S. Adam, and P. W. Brouwer, Phys. Rev. B 74, 134416 (2006).

${ }^{58}$ As expected, this value corresponds to the stray field of the magnetic sphere with magnetic moment $m=2 \times 10^{-10} \mathrm{emu}$ set at a distance $s=1.3 \mu \mathrm{m}$ from the nanopillar.

${ }^{59}$ S. Mizukami, Y. Ando, and T. Miyazaki, Jpn J. Appl. Phys. 40, 580 (2001).

${ }^{60}$ J.-M. L. Beaujour, J. H. Lee, A. D. Kent, K. Krycka, and C.-C. Kao, Phys. Rev. B 74, 214405 (2006).

${ }^{61}$ H. Hurdequint, J. Magn. Magn. Mater. 310, 2061 (2007).

${ }^{62}$ The circular movement is specific to the rotation invariance symmetry. It implies the abscence of harmonic generation which could lead to mode hybridization at high power.

${ }^{63}$ A. G. Gurevich and G. A. Melkov, Magnetization Oscillations and Waves (CRC Press, Boca Raton, FL, 1996).

${ }^{64}$ M. Bailleul, R. Hollinger, and C. Fermon, Phys. Rev. B 73, 104424 (2006).

${ }^{65}$ K. Y. Guslienko, S. O. Demokritov, B. Hillebrands, and A. N. Slavin, Phys. Rev. B 66, 132402 (2002).

${ }^{66}$ It will lead to an error of the order of $4 \pi \gamma M_{s}(t / R)^{2}$ in the frequency, which is much smaller than, for example, the distance between different modes.

${ }^{67}$ J. F. Dillon, J. Appl. Phys. 31, 1605 (1960).

${ }^{68}$ R. W. Damon and J. R. Eshbach, J. Phys. Chem. Solids 19, 308 (1961).

${ }^{69}$ C. Kittel, Phys. Rev. 110, 1295 (1958).

${ }^{70}$ M. Belmeguenai, T. Martin, G. Woltersdorf, M. Maier, and G. Bayreuther, Phys. Rev. B 76, 104414 (2007).

${ }^{71}$ G. Gubbiotti, M. Kostyleva, N. Sergeevaa, M. Conti, G. Carlotti, T. Ono, A. N. Slavin, and A. Stashkevich, Phys. Rev. B 70, 224422 (2004).

${ }^{72}$ J. Ben Youssef and A. Layadi, J. Appl. Phys. 108, 053913 (2010).

${ }^{73}$ O. Dmytriiev, T. Meitzler, E. Bankowski, A. Slavin, and V. Tiberkevich, J. Phys. Condens. Matter 22, 136001 (2010).

${ }^{74}$ [http://www.insilicio.fr/pdf/Spinflow_3D.pdf].
${ }^{75}$ W. E and X.-P. Wang, SIAM J. Numer. Anal. 38, 1647 (2001).

${ }^{76}$ M. d'Aquino, C. Serpico, G. Miano, and C. Forestiere, J. Comput. Phys. 228, 6130 (2009).

${ }^{77}$ R. Lehoucq, D. Sorensen, and C. Yang, ARPACK Users' Guide: Solution of Large-Scale Eigenvalue Problems with Implicitly Restarted Arnoldi Methods (SIAM Publications, Philadelphia, 1998).

${ }^{78}$ R. D. McMichael and M. D. Stiles, J. Appl. Phys. 97, 10J901 (2005).

${ }^{79}$ The two images of Figs. 11(b) and 11(c) are top and bottom view of the nanopillar; hence, one of them should be mirrored for direct comparison of the relative phase between the layers.

${ }^{80}$ M. P. Kostylev, A. A. Stashkevich, N. A. Sergeeva, and Y. Roussigné, J. Magn. Magn. Mater. 278, 397 (2004).

${ }^{81}$ B. Pigeau et al. (unpublished).

${ }^{82}$ S. Kaka, M. R. Pufall, W. H. Rippard, T. J. Silva, S. E. Russek, and J. A. Katine, Nature (London) 437, 389 (2005).

${ }^{83}$ F. B. Mancoff, N. D. Rizzo, B. N. Engel, and S. Tehrani, Nature (London) 437, 393 (2005).

${ }^{84}$ A. N. Slavin and V. S. Tiberkevich, Phys. Rev. B 72, 092407 (2005).

${ }^{85}$ J. Grollier, V. Cros, and A. Fert, Phys. Rev. B 73, 060409 (2006).

${ }^{86}$ B. Georges, J. Grollier, M. Darques, V. Cros, C. Deranlot, B. Marcilhac, G. Faini, and A. Fert, Phys. Rev. Lett. 101, 017201 (2008).

${ }^{87}$ A. Ruotolo, V. Cros, B. Georges, A. Dussaux, J. Grollier, C. Deranlot, R. Guillemet, K. Bouzehouane, S. Fusil, and A. Fert, Nat. Nanotech. 4, 528 (2009).

${ }^{88}$ S. Urazhdin, P. Tabor, V. Tiberkevich, and A. Slavin, Phys. Rev. Lett. 105, 104101 (2010).

${ }^{89}$ A. Dussaux, A. V. Khvalkovskiy, J. Grollier, V. Cros, A. Fukushima, M. Konoto, H. Kubota, K. Yakushiji, S. Yuasa, K. Ando, and A. Fert, Appl. Phys. Lett. 98, 132506 (2011).

${ }^{90} \mathrm{~A}$. Hamadeh et al. (unpublished).

${ }^{91}$ M. Beleggia and M. D. Graef, J. Magn. Magn. Mater. 263, L1 (2003).

${ }^{92}$ S. Tandon, M. Beleggia, Y. Zhu, and M. De Graef, J. Magn. Magn. Mater. 271, 9 (2004).

${ }^{93}$ M. Beleggia, S. Tandon, Y. Zhu, and M. D. Graef, J. Magn. Magn. Mater. 278, 270 (2004).

${ }^{94}$ B. A. Kalinikos and A. N. Slavin, J. Phys. C 19, 7013 (1986).

${ }^{95}$ H. Hurdequint, J. Magn. Magn. Mater. 242-245, 521 (2002). 